\title{
Gradhiva
}

GRADHIV

Revue d'anthropologie et d'histoire des arts

13 | 2011

Pièges à voir, pièges à penser

\section{L'espace chimérique}

Perception et projection dans les actes de regard

Chimeric Space. Perception and Projection in "Gaze Acts"

\section{Carlo Severi}

\section{OpenEdition}

Journals

Édition électronique

URL : http://journals.openedition.org/gradhiva/2021

DOI : 10.4000/gradhiva.2021

ISSN : 1760-849X

Éditeur

Musée du quai Branly Jacques Chirac

Édition imprimée

Date de publication : 18 mai 2011

Pagination : 8-47

ISBN : 978-2-35744-042-5

ISSN : 0764-8928

Référence électronique

Carlo Severi, «L'espace chimérique », Gradhiva [En ligne], 13 | 2011, mis en ligne le 18 novembre 2014 consulté le 19 avril 2019. URL : http://journals.openedition.org/gradhiva/2021 ; DOI : 10.4000/ gradhiva.2021

(c) musée du quai Branly 


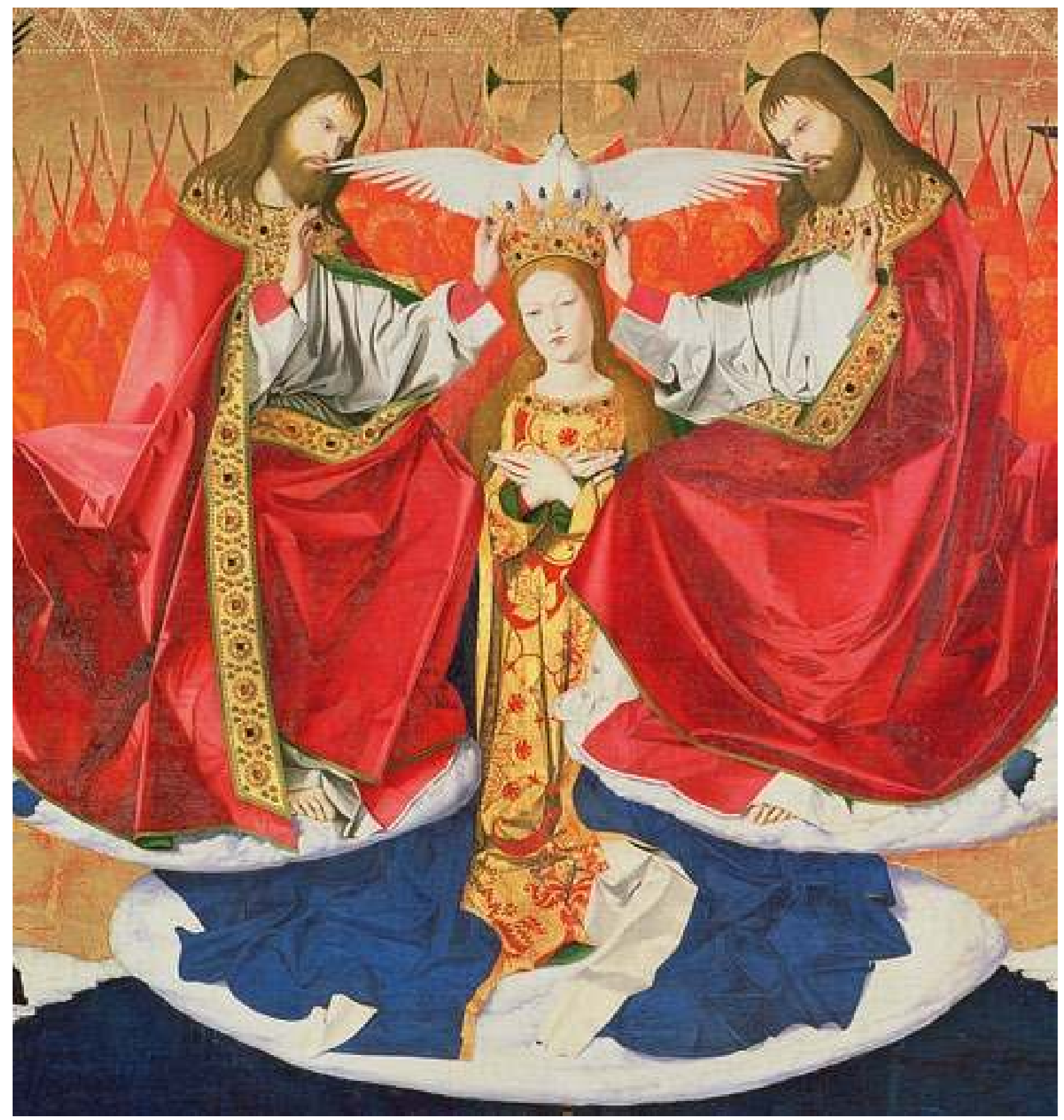

Fig. 1 Enguerrand Quarton, Couronnement de la Vierge, détail, 1454, huile sur panneau. Musée Pierre de Luxembourg, Villeneuve-lès-Avignon (๑) musée Pierre de Luxembourg/Giraudon/The Bridgeman Art Library. 


\section{L'espace chimérique Perception et projection dans les actes de regard}

J'ai vu l'image de tout un paysage se réfracter dans l'œil de l'échassier lorsqu'il plonge dans l'eau : les mille cercles qui enserrent chaque vie, le bleu du ciel chuchotant avalé par le lac, l'émergence en un autre lieu - voilà ce que sont les images : l'émergence en un autre lieu.

Franz Marc (1996 [1914])

Carlo Severi
Le musée Pitt Rivers d'Oxford possède une singulière boucle en ivoire, collectée en Sibérie (fig. 2). On y voit deux formes identiques interprétées de manière différente : une fois comme le contour d'une tête de loup, et une autre fois comme celui d'un corps entier, probablement celui d'un lion de mer. Cet objet ne constitue pas uniquement la représentation de deux animaux différents par les mêmes moyens visuels : il témoigne d'un acte de regard. En insérant une ligne courbe dans deux contextes différents, l'image passe de la représentation par imitation d'un animal, à l'interprétation, complexe et plurielle, d'une forme. En tant que trace matérielle, la représentation fait émerger un travail de l'esprit, une série d'opérations mentales (ou même, plus simplement, de pensées) qui se trouvent associées à une ligne, et qui en font surgir, dans les deux cas, une partie invisible, ou potentielle.

Il y a quelques années (Severi 2003), nous avons proposé d'appeler chimérique ce type de représentation, en soulignant que ce qui la caractérise est la condensation de l'image en quelques traits essentiels. Cette condensation engendre, par projection, une ou plusieurs interprétations de la forme. Ce qui est donné à voir est implicitement considéré comme une partie d'une autre forme, dont la présence est imputée, et éventuellement représentée. Dans un tel acte de regard, l'invisible prime sur le visible, et semble en fournir le contexte. Nous avons formulé l'hypothèse que cette structure "par indices" confère à l'image une saillance particulière qui lui permet de jouer un rôle crucial dans les pratiques sociales liées à la mémorisation et à la mise en place d'un savoir dans un certain 

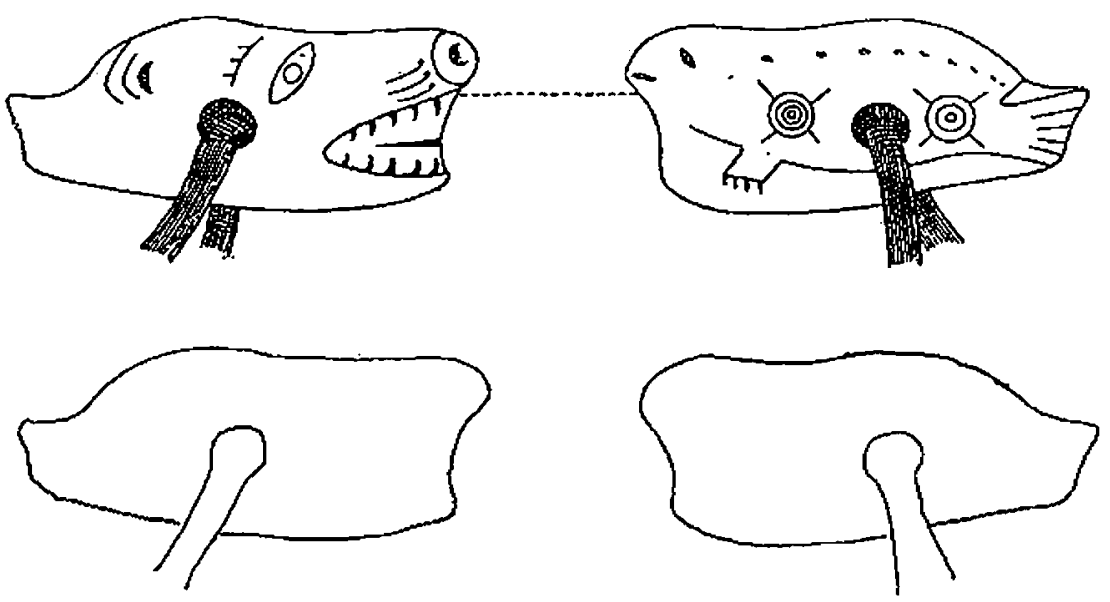

Fig. 2 Boucle sibérienne en ivoire de lion de mer, musée Pitt Rivers, Oxford.

nombre de traditions habituellement appelées «orales». La saillance visuelle de ces images, liée à la mobilisation de l'inférence qu'elles impliquent, peut devenir ainsi saillance mnémonique, capacité de véhiculer et préserver du sens.

Cette première définition, qui visait à rendre compte du mode de fonctionnement d'un certain nombre d'exemples spécifiques dans le cadre d'une théorie anthropologique de la mémoire, a suscité autant de débats que de recherches nouvelles. Parmi les questions qui ont été soulevées, un premier groupe concerne l'interprétation de l'ethnographie: comment apprécier, sur de nouveaux terrains, la capacité heuristique de la notion de chimère? Peut-on, à partir de ces premières analyses, définir un type général de représentation, qu'on pourrait appeler "chimérique" comme on parle, par exemple, de représentations "réalistes", "abstraites", ou "symboliques"? Comment identifier des modes de variation, dans l'espace ou dans le temps, des représentations chimériques? Telle qu'elle se trouve définie à partir de ces premiers exemples, la représentation "chimérique » serait-elle propre aux arts non occidentaux? Si tel était le cas, comment différencier ce qui serait propre aux chimères amérindiennes, océaniennes ou africaines, de tout ce que la tradition occidentale a connu sous le nom d'image fantastique, double, ambigüe ou même, selon la récente définition de Dario Gamboni (2004), simplement «potentielle »?

D'autres questionnements concernent les aspects plus proprement logiques de cette notion : si l'essentiel de l'idée de chimère ne concerne pas un type d'image défini par une morphologie spécifique, mais plutôt le type d'opérations mentales que l'invention et l'appréhension de ce type d'image implique (sélection des traits visuels, projection, induction, établissement de séquences, etc.), qu'est-ce qui est propre, de ce point de vue, à la représentation chimérique? Quel exercice de la pensée la caractérise, et permet éventuellement de l'opposer à d'autres modes de pensée?

D'autres questions, enfin, ont été formulées d'un point de vue plus proche de l'esthétique. On admettra volontiers, dans cette perspective, qu'un des effets de la représentation chimérique est d'intensifier une image grâce à la mobilisation de ses aspects invisibles. Mais au fond, pourrait-on objecter, toute œuvre d'art suscite, depuis toujours, un travail de la pensée qui concerne ce qui n'y est pas matériellement représenté. Tous les classiques de la pensée esthétique moderne 
ont souligné ce point. Dans son Essai sur la peinture, Diderot, décrivait déjà, avec une grande précision, ce jeu de regard, cette machine qui fait surgir, à travers un calcul attentif de l'espace et des proportions, la part d'invisible d'un tableau :

Tâchez mes amis - écrivait-il en s'adressant en particulier aux dessinateurs - de supposer toute la figure transparente, et de placer votre œil au centre : de là vous observerez tout le jeu extérieur de la machine; vous verrez comment certaines parties s'étendent, tandis que d'autres se raccourcissent; comment celles-là s'affaissent, tandis que celles-ci se gonflent; et perpétuellement occupés d'un ensemble et d'un tout, vous réussirez à montrer, dans la partie de l'objet que votre dessin présente, toute la correspondance convenable avec celle qu'on ne voit pas, et, en n'offrant qu'une face, vous forcerez mon imagination à voir encore la face opposée; c'est alors que je m'écrierai que vous êtes un dessinateur surprenant. (1951: 1118)

Comment préciser, de ce troisième point de vue, ce qui est propre à l'appréhension esthétique de la représentation chimérique? Et comment concevoir, si toute œuvre d'art suppose une réflexion, une image qui ne serait pas chimérique?

Tenter de répondre à ces questionnements peut nous permettre non seulement d'affiner nos outils d'analyse (et d'éclaircir parfois quelques malentendus), mais aussi de renouveler notre compréhension du type d'exercice de la pensée qui s'exprime au sein d'une tradition iconographique. On passera alors de la perspective qui conduit à définir une typologie des représentations, à l'identification d'une logique des relations représentées par l'image au sein d'une tradition. Pour montrer comment il est possible d'opérer ce changement de perspective, nous essaierons d'abord de préciser la définition et les développements possibles de la notion de chimère à partir des trois points de vue que nous avons identifiés : morphologique, logique et esthétique. Dans un second temps, nous essaierons de montrer comment cette nouvelle approche permet d'interpréter un cas ethnographique précis.

Mais reprenons les questionnements que le débat sur la représentation chimérique a suscités, et considérons d'abord la question, posée du point de vue esthétique, de la singularité du chimérique par rapport à la relation qui s'établit entre œuvre d'art et travail de la pensée.

«L'artiste doit savoir offrir plus à l'esprit qu'au regard... le propre de la peinture est de pouvoir représenter des choses invisibles, qui se situent dans le passé ou dans le futur. » Winkelmann, Réflexions sur l'imitation de l'art des Grecs (1973).

Dans cet aphorisme, Winkelmann formule un des fondements de la pensée esthétique moderne : loin de mobiliser un processus de perception passif ou mécanique, l'œuvre de l'artiste suscite toujours chez l'observateur un acte de regard. Dans un esprit proche de celui de Diderot, Winkelmann affirme que ce qui surgit au sein de l'expérience esthétique est le fruit d'un dialogue entre ce qui est donné à voir sur une toile peinte et un processus d'exploration/interprétation où l'observateur joue un rôle parallèle à celui de l'auteur. Dans cette perspective, l'œuvre dépasse, et même s'oppose à l'image. À partir de ce qu'il voit, le sujet qui se constitue en observateur fait émerger une expérience esthétique qui, bien que partiellement imaginée par l'artiste, ne se réalise pleinement qu'au sein du regard de l'observateur. De Goethe, qui affirmait que «le monde extérieur n'a pas de couleur : seule l'étincelle de lumière qui réside dans l'œil confère au monde son chromatisme » (2000 [1808: 21]) à Lévi-Strauss qui, dans La Pensée sauvage, a pu parler d'un observateur « qui se 
DOSSIER

PIÈGES À VOIR, PIÈGES À PENSER

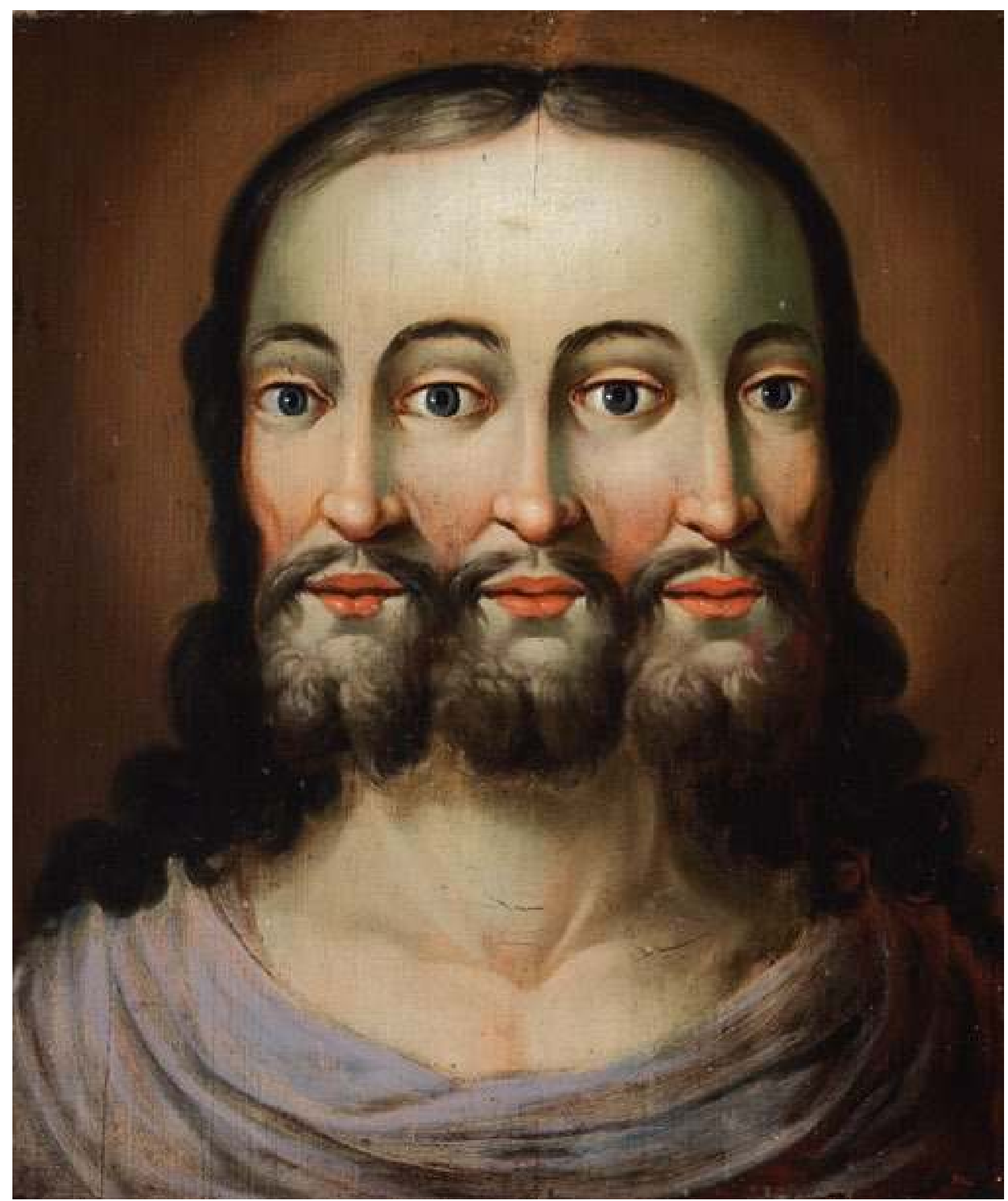

Fig. 3 Anonyme, La Trinité, xvIII siècle, huile sur bois $(43,5 \times 28,5$ cm). Museum Carolino Augusteum, Salzbourg. 
sent confusément créateur à meilleur titre que le créateur lui-même " (2008:586), cette idée de l'œuvre comme le résultat d'un dialogue engagé à travers un acte de regard (et non pas par la simple perception visuelle) a été développée par toute une tradition de pensée. Dans L'Eil du Quattrocento, Baxandall (1985) en formule une version particulièrement claire, qui peut servir d'introduction aux analyses que nous allons proposer dans cet article. Dans un bref chapitre consacré à la relation entre expérience optique et connaissance culturelle, l'historien anglais propose une sorte d'expérimentation visuelle. Soit la figure 4. Que représente-t-elle? On peut y voir par exemple une forme ronde, assez vaguement tracée, flanquée de part et d'autre de projections allongées, en forme de L. Ou bien, d'un point de vue plus géométrique, un cercle plaqué sur un rectangle tronqué. La perception qu'on pourra en avoir dépendra non seulement du processus mécanique qui règle la perception visuelle, mais aussi « des capacités interprétatives, des catégories, des modèles et des habitudes de déduction et d'analogie " qui forment «ce qu'on peut appeler le style cognitif» d'un observateur donné (Baxandall 1985 : 48). Savoir que cette image provient d'une description de la Terre sainte publiée à Milan en 1481,

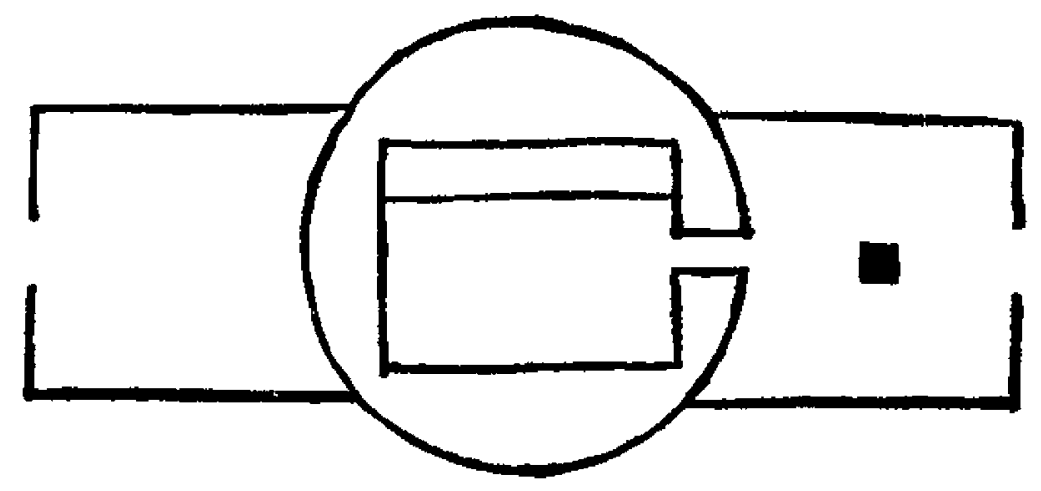

et qu'elle est accompagnée de la légende «Ceci est la forme du Saint-Sépulcre de Notre Seigneur Jésus-Christ » apporte deux éléments importants à la perception de cette image. D'abord, selon Baxandall, l'observateur pourra se référer à l'expérience qu'il peut avoir d'un certain nombre de conventions représentationnelles. Il ou elle pourra alors juger que cette image relève de la projection plane, une convention de lecture selon laquelle les lignes qui représentent les murs périphériques d'un édifice se dessinent sur le sol si on le regarde verticalement, vu d'en haut. Ensuite, si l'on est familier de l'architecture italienne $d u x^{e}$ siècle, on pourra déduire du dessin que le cercle représente ici un bâtiment circulaire, peut-être surmonté d'une coupole, que les ailes rectangulaires sont des vestibules, et que le carré à l'intérieur du cercle désigne l'espace où se trouve la tombe (ibid. : 49). Trois facteurs variables, liés à la culture, agissent donc sur la façon dont notre esprit interprète ces formes, qui ont pu paraître, jusque-là, vides de sens : «un fonds de modèles, de catégories et de méthodes déductives; l'entraînement à une série de conventions pour représenter les choses et, enfin, l'expérience des manières plausibles de visualiser ce sur quoi nous n'avons qu'une information incomplète " (ibid.). On en conclura que dans tout acte de regard, tel qu'il s'exerce dans une culture donnée, la perception en tant que processus physiologique et la projection d'un certain nombre de savoirs acquis se trouvent strictement associées. En tant qu'opérations mentales, elles sont indissociables de tout acte de regard. Toutefois, cela ne signifie pas que cette relation entre perception et projection, qui définit le processus de construction culturelle
Fig. 4 Santo Brasca, plan du Saint-Sépulcre à Jérusalem, Itinerario alla Santissima città di Gerusalemme. Milan, Leonardus Pachel et Uldericus Scinzenzeler, $1481: 58 \mathrm{v}$. 
d'une image, s'établit toujours dans les mêmes conditions, comme si les conventions visuelles auxquelles se réfère ici Baxandall constituaient une sorte de grammaire de la culture, dont les règles seraient faciles à énoncer, ou même fixées à jamais. Instable, variable de contexte à contexte, liée à l'hypothèse ou à l'inférence, toujours à reconstruire, cette relation entre perception passive et regard interprétatif peut varier non seulement d'individu à individu, mais aussi selon le type de dialogue qu'une convention visuelle (ou l'ensemble d'une tradition iconographique) propose au regard d'un observateur. Si nous voulons comprendre ce qui, de ce point de vue, est propre à la représentation chimérique, il faudra donc comprendre quelle relation entre projection et perception ce type de représentation implique, et selon quelles modalités. Pour comprendre les chimères, il faudra essayer d'identifier les coordonnées de l'espace chimérique. Mais considérons d'abord la convention visuelle qui nous est la plus familière, celle de la perspective occidentale.

II

La relation la plus simple entre perception et projection est sans doute celle d'enchâssement, qui conduit à inscrire l'exercice d'une opération dans le cadre conceptuel fourni par l'autre. La projection s'exerce alors dans un cadre visuel virtuellement fixe, comme peutl'être, par exemple, celuidela perspective occidentale. Dans ce cadre, projeter signifie, avant même de commencer à déchiffrer la signification d'une image, devenir capable de traduire des indications statiques disposées sur une surface, en indications de profondeur douées d'un mouvement implicite. Ce type d'appréhension de l'image, qui nous semble si familier, est loin d'être universel. Il a été analysé en détail, en tant que dispositif culturel de fonctionnement optique, notamment par Florensky (1992). Mais c'est sans doute dans le Problème de la forme d'Hildebrand (2001 [1893]) que l'on trouve, d'un point de vue formel, la description la plus claire de ce processus de déchiffrement de la profondeur à travers la perception d'images disposées sur une surface plane - auquel l'existence même de la perspective en tant que convention visuelle nous a habitués.

Suivons son raisonnement : l'œuvre d'art suscite le travail de la pensée, écrit Hildebrand, parce que, loin de se fonder sur l'observation directe du réel, elle résulte d'un processus complexe de remémoration de l'image réelle :

«Voir et représenter un objet sont deux processus très différents : pour en avoir la preuve, regarde un objet attentivement, et tourne-lui le dos. Ce qui reste dans ton esprit est très différent de la première impression que tu en as eue : une partie de l'image a disparu, d'autres traits persistent. Le premier acte est une perception, le deuxième est une représentation. L'acte qui préside à la représentation est donc propre au souvenir, et non à la perception. » (2001 [1893] : 122)

La mémoire visuelle est donc, à proprement parler, la «matière mentale " sur laquelle l'artiste intervient. Son travail implique bien, selon l'expression d'Hildebrand, une constante «évaluation des apparences ». Mais cette attention au réel n'a pas pour objet l'imitation de la nature. Elle vise à l'identification d'un principe organisateur de la perception, qui s'exprime par la présence d'une forme. Ce concept ne désigne, pour Hildebrand, ni un phénomène propre à l'apparence du monde extérieur, ni un aspect de l'expérience qui appartiendrait seulement à l'activité artistique. Le sentiment de la forme renvoie pour lui à «la perception inconsciente de l'espace qui oriente constamment notre regard ». Ce "sentiment " (qu'on appellerait plus volontiers, de nos jours, un réflexe inconscient) oriente constamment nos mouvements, et marque la présence de notre corps dans l'espace. Dans ce cadre, 


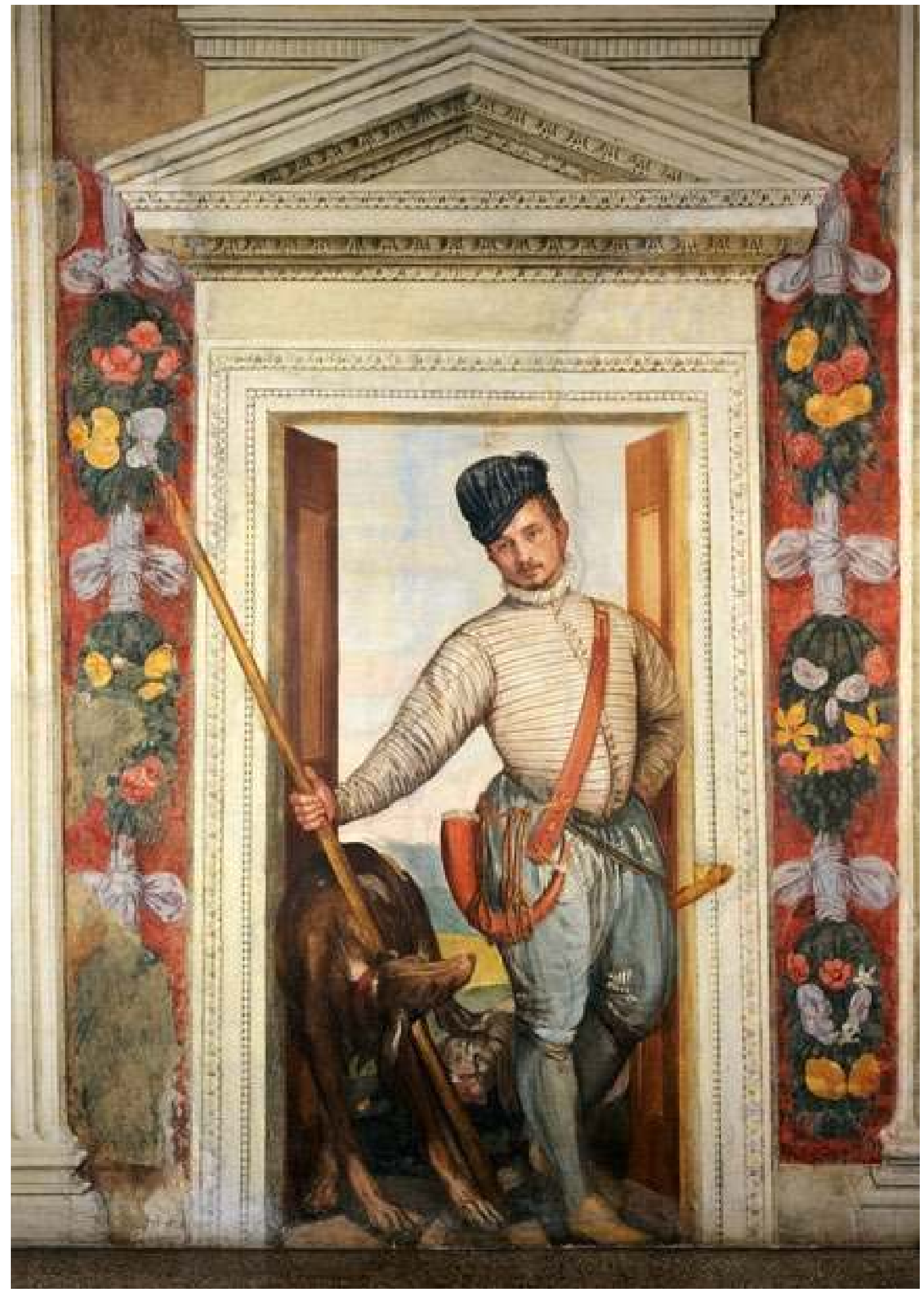

Fig. 5 Véronèse (Paolo Caliari), Présumé autoportrait en chasseur, Fresque de la villa Maser, détail, 1560-1561, Vicence @ 2011. Photo Scala, Florence. 


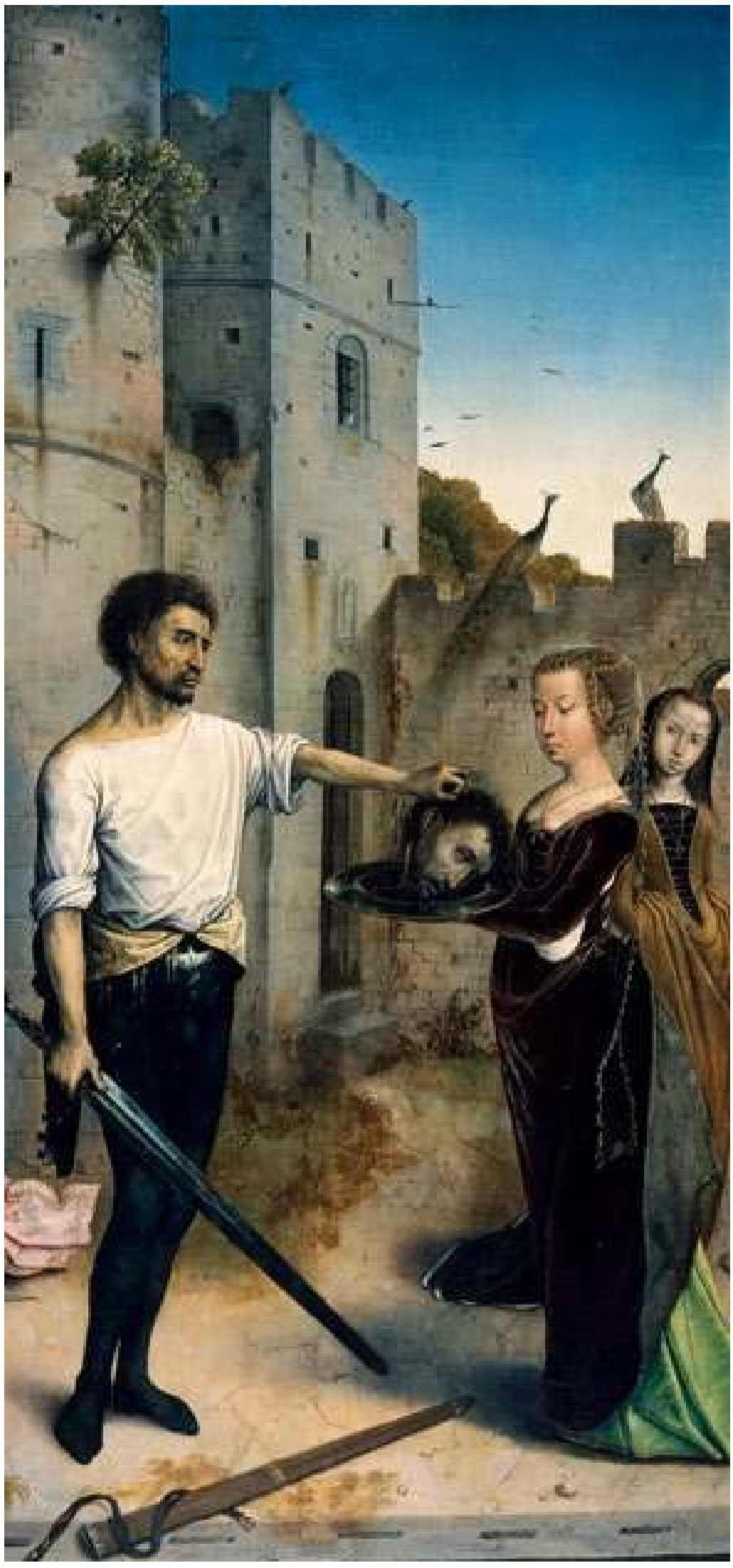

toute image qui, comme dans la convention visuelle de la perspective, donne à voir la profondeur et le mouvement implicite, a pour objet non pas l'imitation de l'apparence, mais la représentation de l'espace à travers le travail du regard. Hildebrand en conclut que l'artiste travaille toujours «là où les représentations de l'espace sont inconsciemment produites" (ibid. : 228). Atteindre ce substrat, le rendre sensible, c'est tout autant explorer le travail du regard que susciter le sentiment inconscient de l'espace.

Il n'est pas nécessaire de reprendre ici d'autres aspects de la conception d'Hildebrand de la forme. Retenons-en seulement un point essentiel : sa définition offre un modèle, à la fois abstrait et élémentaire, de la relation d'enchâssement entre perception et projection qui se réalise au sein de la convention visuelle de la perspective. Au sein de cette convention, devenue dominante en Occident, on ne peut saisir la forme (et donc, interpréter correctement la perspective) qu'en faisant surgir, à partir d'indications disposées sur une surface, la profondeur et le mouvement implicite qui caractérisent une représentation.

Fondateur du formalisme, plus théoricien de l'espace esthétique qu'historien de l'art, Hildebrand ne donne qu'une interprétation idéalisée des relations qui peuvent s'établir dans ce cadre entre perception et projection. En réalité, l'histoire des pratiques liées à la perspective est loin d'être régie seulement par des règles de géométrie. Une fois ce cadre fixé selon le modèle albertien, ces pratiques ont progressivement donné naissance à un espace partagé, avec ses permanences, ses problèmes typiques, ses dilemmes, ses retrouvailles et ses résonances, dont l'évolution complexe se situe sans doute dans la longue durée. En poursuivant sur ce point la réflexion de Baxandall et de Shearman (1992), Svetlana Alpers (2005) a comparé cet espace à un laboratoire scientifique où l'artiste, son modèle, et son commanditaire jouent, chacun de 
son côté, un rôle essentiel. À l'intérieur de cet univers, (une sorte de théâtre du regard, où, du Quattrocento aux impressionnistes, tout élément de la scène peut varier : l'idéologie du commanditaire, les techniques de la représentation, l'attitude du modèle, ou même, comme l'a montré Fried [1990], sa manière de s'adresser à l'observateur) plusieurs modalités de relation entre projection et perception sont possibles. Mais prenons ici le risque de simplifier : le moyen le plus simple de mobiliser la projection pour interpréter une image inscrite sur une surface plane consiste, dès la Renaissance, à lui conférer une valeur symbolique. Baxandall $(1985,1989)$ et Settis (2005) ont montré par exemple que toute l'iconographie religieuse de la Renaissance italienne est rigoureusement codée à partir d'instructions détaillées que l'Église a dictées aux artistes afin d'orienter et exercer l'imagination des fidèles. On retiendra ici l'exemple de la représentation du Christ, dont Baxandall a montré qu'elle n'était nullement abandonnée à l'imagination. Sa figure devait suivre la description donnée dans un compte-rendu que l'on supposait envoyé par Lentulus, gouverneur de Judée, au Sénat romain. Selon Lentulus (sans doute un personnage légendaire), qui était censé avoir connu le Christ, celui-ci était « un homme de taille moyenne ou petite... ses cheveux étaient de couleur noisette mûre et descendaient droit jusqu'à la hauteur des oreilles pour tomber ensuite en boucles épaisses jusqu'aux épaules... son front était vaste, poli et serein, son visage dépourvu de rides, sa barbe, de la même couleur que les cheveux, ressemblait à la première barbe d'un jeune homme ».
PAGE CI-CONTRE

Fig. 6 Juan de Flandes,

La Décollation de saint Jean-

Baptiste, détail, vers 1496-1499

(c) musée d'Art et d'Histoire,

Ville de Genève, inv. no CR 365/

Photo Bettina Jacot-Descombes.

Fig. 7 Albrecht Dürer, Hiéroglyphes pour l'arc de Triomphe de l'empereur Maximilien, vers 1515 . Collection privée/

The Bridgeman Art Library.
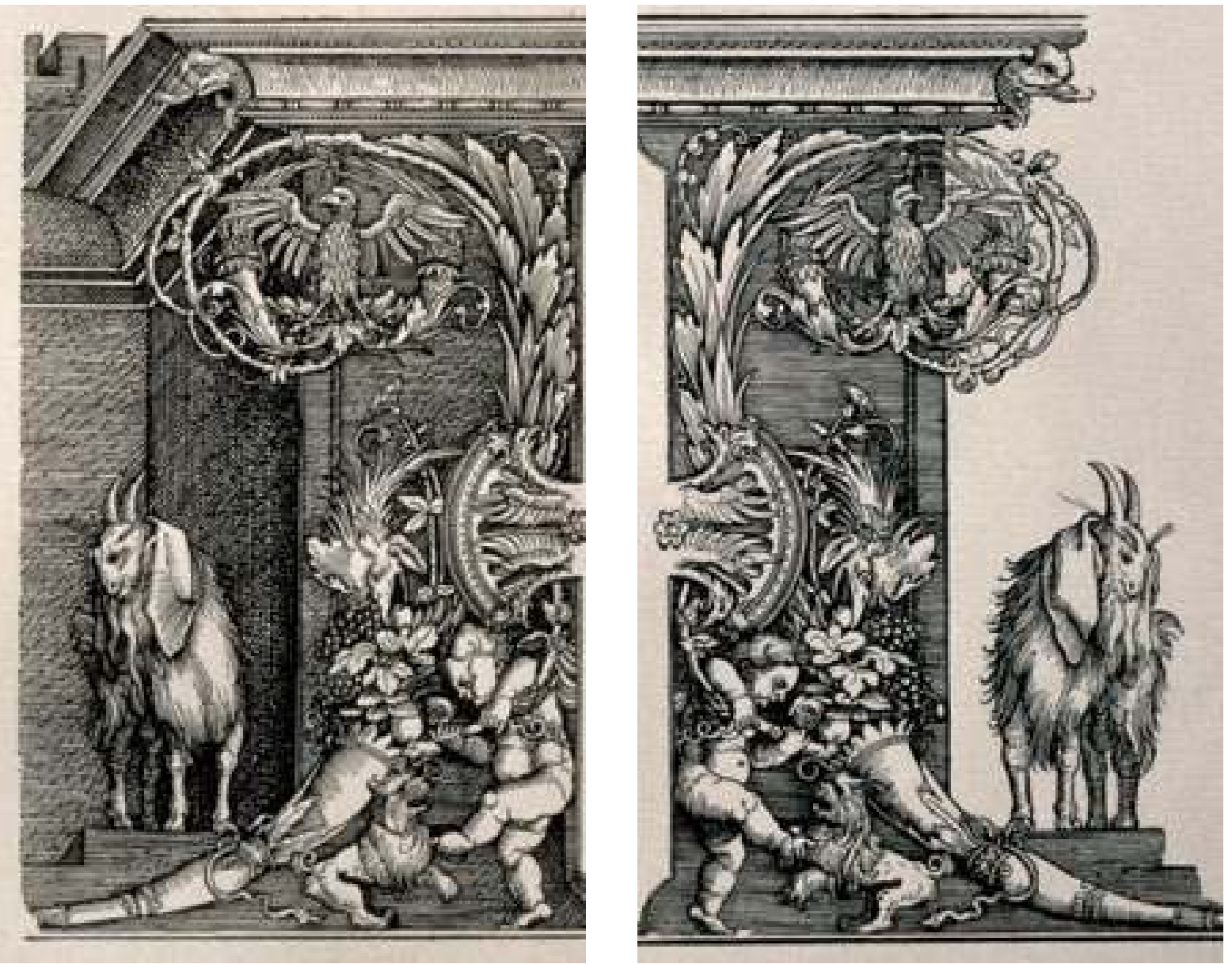
PIÈGES À VOIR, PIÈGES À PENSER

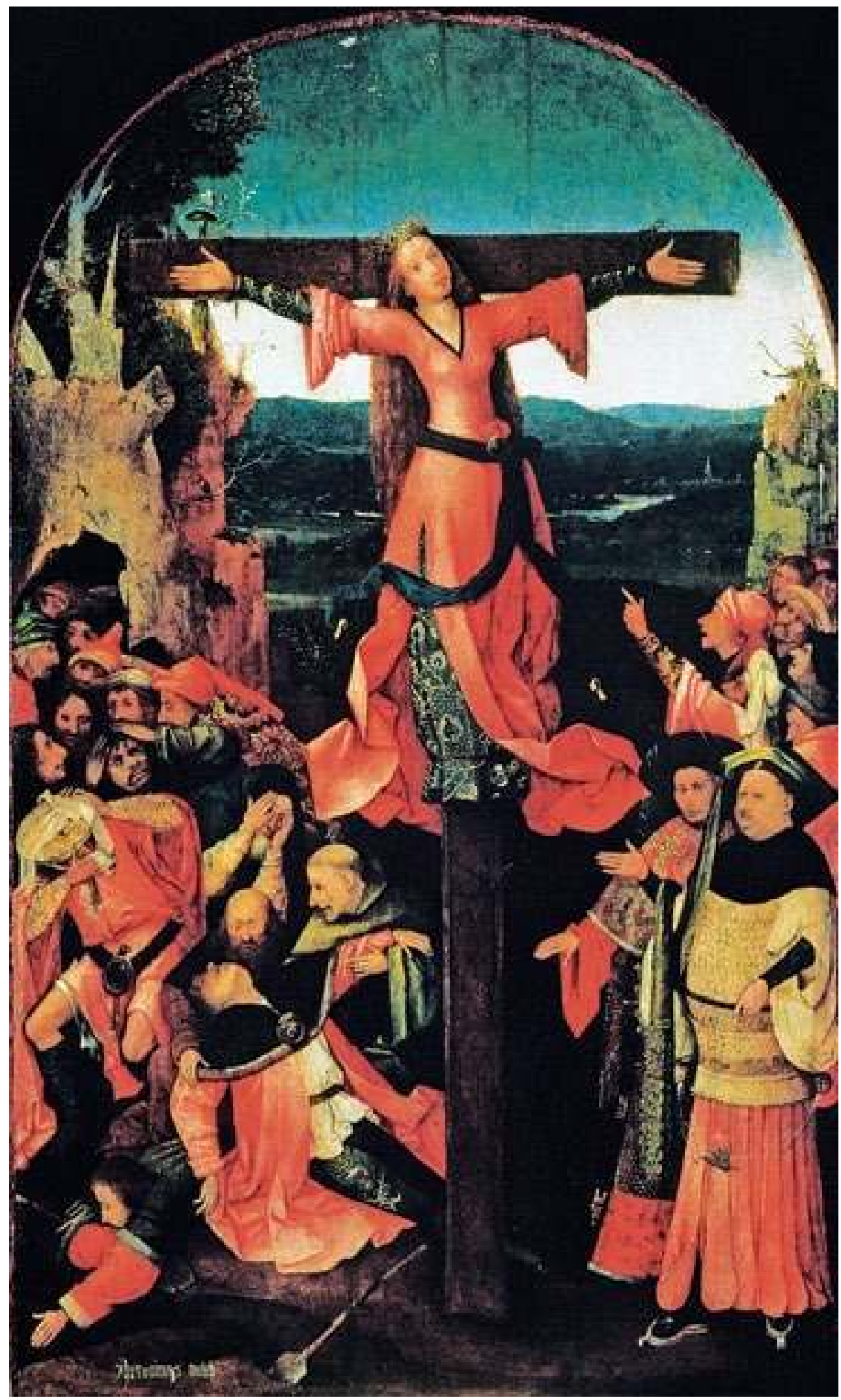

Fig. 8 Jérôme Bosch, Le Martyre de sainte Liberata, huile sur panneau (104 x 119 cm). Palais des Doges, Venise. (c) Palazzo Ducale, Venise/The Bridgeman Art Library. 
Peu de peintures, en effet, contredisent ce modèle (Baxandall 1985: 91). On soulignera toutefois qu'à l'intérieur de ce cadre iconographique, il était tout à fait admis que l'aspect conceptuel d'une représentation puisse contredire sa vraisemblance. Restons-en à la représentation de Jésus-Christ en jeune homme : dans un Couronnement de la Vierge peint vers 1454, près d'Avignon, Enguerrand Quarton, pour montrer qu'il ne peut y avoir de différence entre le Père et le Fils, les représente par deux visages identiques (fig. 1) (Baschet 2008: 170-172). De manière analogue, Bramantino peint, dans les mêmes années, une Vierge à l'enfant dont les traits sont si proches de ceux d'un jeune homme, que le tableau a longtemps paru très énigmatique. Suida a montré qu'il s'agissait d'une part, pour les commanditaires de l'œuvre, de rappeler la nature divine du Christ, qui peut ainsi contredire les apparences et surgir à travers le visage de sa mère, et d'autre part d'évoquer, à travers la ressemblance de la mère au Fils, une définition canonique, mais rare, de la Vierge en tant que «Vierge Mère, Fille de son Fils » (Vergine madre, figlia del tuo figlio, dira Dante dans le chant XXXIII du Paradis) (Suida 1953 : 98-100). On retrouve les mêmes jeux de «ressemblance impossible » chez Bosch, lorsqu'il donne à sainte Liberata, sacrifiée sur la croix, le visage du Christ (fig. 8). Plus tard, un anonyme allemand du xviII siècle (en s'inscrivant dans une tradition fort ancienne) n'hésitera pas à représenter la Trinité par un triple visage de jeune homme (fig. 3).

La référence à l'Antiquité grecque, latine ou égyptienne a naturellement produit une iconographie conventionnelle fort influente, dont toute l'école de Warburg a développé l'analyse, et sur laquelle nous n'insisterons pas ici. On remarquera toutefois, dans l'esprit de Baxandall, que ces données iconographiques de base ne constituent qu'un répertoire relativement stable, que l'on pourra, selon les occasions, citer, modifier, contredire, ou renouveler. Qu'on pense aux «hiéroglyphes » dessinés par Dürer autour de 1515 pour l'arc de Triomphe de l'empereur Maximilien (fig. 7), où toute une tradition iconographique néo-égyptienne (Barasch 2003, Panofsky 1943, Wittkower 1977) est complètement réinventée, aussi bien en ce qui concerne le style que la signification des symboles. Au-delà de la tradition chrétienne, ces usages de l'image symbolique semblent bien s'organiser, comme l'a reconnu Warburg (2010), selon deux axes : ou bien ils renvoient au retour de l'Antique (selon l'usage italien), ou bien ils se réfèrent à la tradition alla francese que Warburg avait identifié en étudiant les tapisseries bourguignonnes des collections des Médicis à Florence (ibid. : 5). Cette manière française (très tôt reprise dans toute l'Europe, y compris en Italie) consiste à situer des scènes tirées d'un texte antique, de l'Évangile ou de la Bible, à une époque contemporaine à celle de l'artiste et de son commanditaire. La Décollation de saint Jean-Baptiste de Juan de Flandes (fig. 6), peinte autour de 1496-1499, offre un exemple saisissant de cette manière novatrice de faire varier le symbolisme. Le maître flamand (qui fait allusion à un texte de Matthieu où on parle de la beauté sensuelle de Salomé, et de la cruauté qu'elle a montrée lorsqu'elle a demandé à Hérode la décapitation de saint Jean-Baptiste) nous montre la jeune princesse accompagnée d'une dame de sa cour. Habillée, comme sa compagne, dans un costume d'une grande élégance, Salomé semble parfaitement indifférente, face au garde qui, plus troublé, lui tend la tête décapitée de Jean-Baptiste. Plus loin, posés sur le mur d'enceinte du château, deux merveilleux paons apparaissent. En contre-jour d'une lumière dorée d'après-midi, les oiseaux paraissent élégants, indifférents et cruels, autant que les dames. Cette première analogie acquiert une intensité singulière lorsque l'observateur s'aperçoit que le peintre 
fait jouer, face à l'axe dames/oiseaux, un autre axe analogique, en donnant à la tête du garde et à celle du saint décapité des traits, marqués par la douleur, que l'on ne peut s'empêcher de trouver presque identiques. L'incarnation d'une cruauté maîtrisée, sans émotion apparente, fait ici face à une représentation si intense de la souffrance, qu'elle en devient presque hallucinatoire. Il serait sans doute difficile de trouver un exemple plus éloquent du style alla francese : entièrement confié à l'invention d'images, le texte et son symbolisme sont là, sans que rien ne renvoie explicitement à l'Évangile.

III

Inspiré par l'Antiquité, par la tradition religieuse, ou par les usages de la vie de cour, le symbolisme témoigne donc du travail de projection de différents savoirs, qui, à la Renaissance, s'associent à l'interprétation visuelle d'un tableau. Mais ce travail de la projection (cet acte de regard qui, face à l'œuvre, mobilise un savoir acquis) que nous avons vu jusqu'à présent opérer au niveau sémantique, peut aller au-delà du déchiffrement des images symboliques, et investir l'espace même de la représentation. Le cadre formel de la perspective (et le type d'articulation qu'il suppose entre perception et projection) cesse alors d'apparaître comme une donnée à reproduire mécaniquement, et tend à acquérir un aspect réflexif. L'hypothèse sur laquelle repose la perspective en tant que convention visuelle, à savoir l'existence d'une continuité entre l'espace peint et l'espace réel (et en particulier de cette partie de l'espace réel qu'on peut appeler liminale, puisqu'elle « désigne la frontière de l'espace représenté sans en faire partie » [Shearman $1992: 59$ ]), cesse de fonctionner comme un implicite, et émerge comme sujet de la représentation.

Le regard de l'observateur passe ainsi du déchiffrement des significations symboliques des figures à l'interprétation de l'action représentée, et de l'espace qu'elle implique. Une série de gestes, de regards et de postures apparaissent, supposant l'existence d'un espace mi-fictif, mi-réel, dans lequel celui qui regarde se trouve capté. On découvre ainsi ce que Shearman, en reprenant la définition classique de Riegl (2009 [1902]) et les travaux de Gombrich (1969, 1982), a appelé la transitivité de la perspective. Il s'agit de sa capacité, toute formelle, à susciter la présence d'un observateur engagé dans l'image. En tant que convention visuelle, celle-ci implique non seulement l'existence d'un "point de fuite» qui organise en un espace cohérent la perception de la profondeur, mais aussi celle de deux foyers visuels : l'un situé à l'intérieur du tableau, et l'autre qui se projette à l'extérieur de l'espace peint, et marque ainsi la place implicite de l'observateur (Shearman 1992 : 36).

Lorsque le travail de la projection prend pour objet le cadre de la représentation, l'image inclut dans l'espace fictif qu'elle donne à voir certains éléments de l'espace réel où elle est située. Elle représente ainsi, pour ainsi dire en ellemême, les conditions de sa propre perception. Cette mise en abîme fait émerger

1. Le niveau d'analyse que Karl Bülher a appelé, dans sa théorie du langage (1990 [1934]), le MOI-ICI-MAINTENANT de l'énonciation, deviendrait ainsi pertinent pour la représentation visuelle, ce qui permettrait de renouveler l'interprétation de l'agentivité (au sens de Gell [1998]) attribuée à l'image (Severi 2010). ce qu'on pourrait appeler les aspects pragmatiques de l'acte de regard'. Ce type de composition (qui inclut dans la scène donnée à voir ses conditions de perception visuelle) marque une relation nouvelle entre l'exercice de la perception visuelle et celui de la projection. On a vu qu'au sein de la perspective, elles s'articulaient par enchâssement. Or, ce rapport implique que l'aspect conventionnel de la vision, qui fonctionne comme cadre de l'activité d'interprétation, ne soit jamais représenté en tant que tel. Lorsque le spectateur traduit des indications disposées sur une surface plate, par projection, en termes de profondeur et de mouvement implicite, les coordonnées formelles du cadre, qui orientent la perception, 


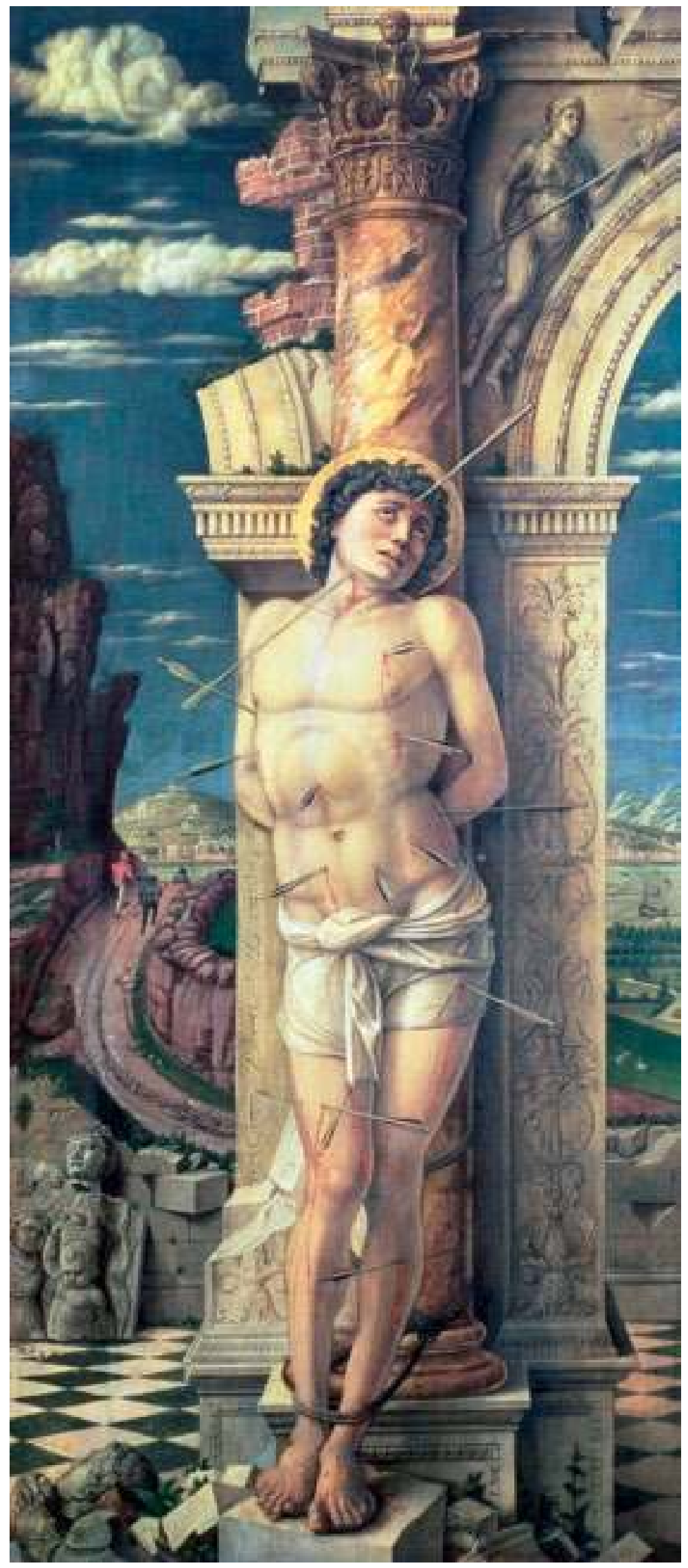

Fig. 9 Andrea Mantegna,

Saint Sébastien, vers 1459,

tempera sur panneau

$(68 \times 30 \mathrm{~cm})$. Kunsthistorisches Museum, Vienne.

(c) Kunsthistorisches Museum

/Ali Meyer/The Bridgeman Art Library. 
PIÈGES À VOIR, PIÈGES À PENSER

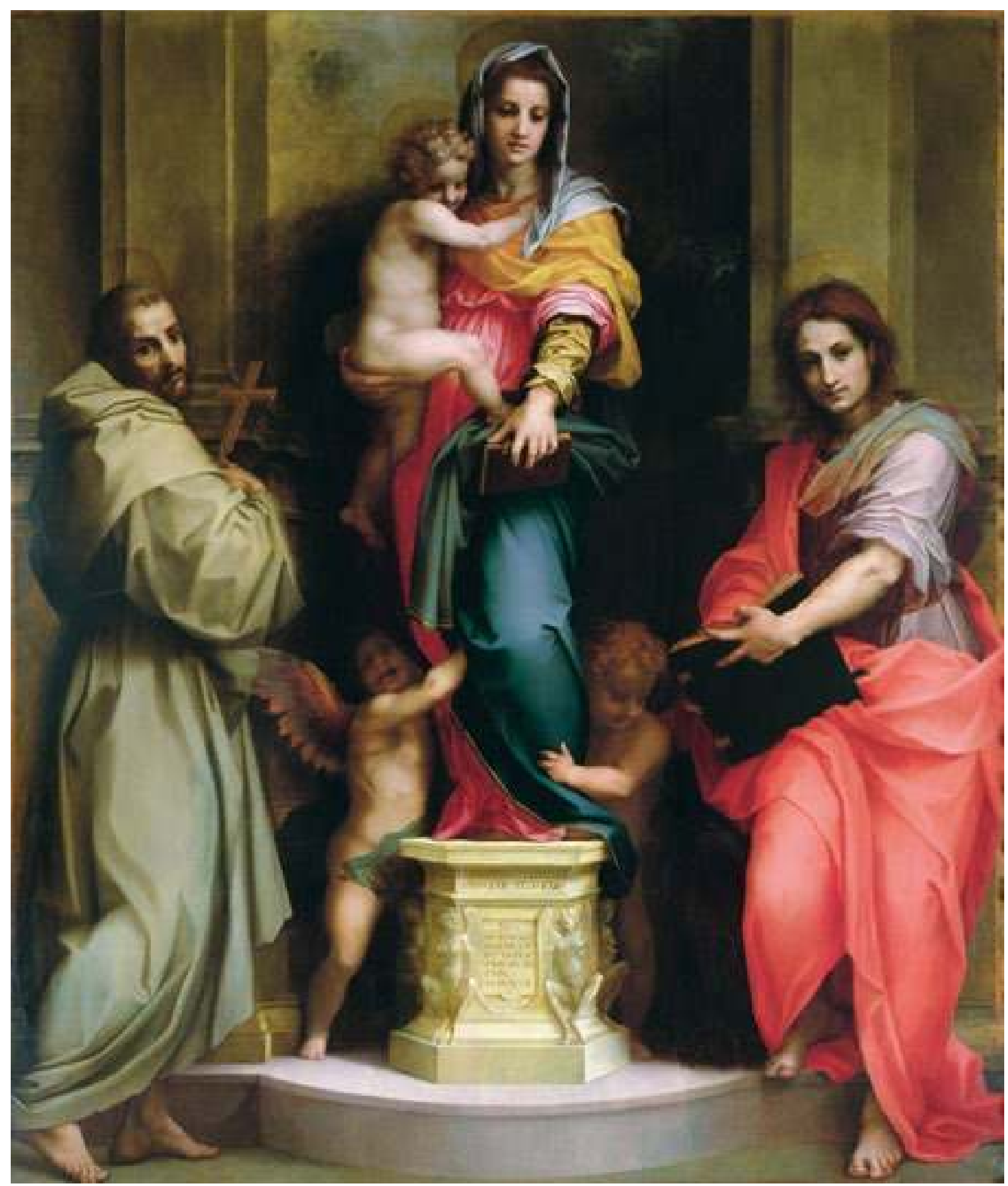

Fig. 10 Andrea Del Sarto, Vierge aux harpies, 1517, huile sur panneau (178 × 207 cm). Galerie des Offices, Florence $\odot$ Galleria degli Uffizi/ The Bridgeman Art Library. 
disparaissent de la conscience. Le "sentiment de la forme", dans la convention visuelle qui rend possible la perspective, s'avère bien tel qu'Hildebrand l'avait défini : "un réflexe inconscient de l'espace qui oriente inconsciemment la position du corps et le regard" (2001 [1893] : 228). Il va sans dire que ce type d'enchâssement opère, a fortiori, lorsque le regard de l'observateur interprète une représentation symbolique, dont la perception de la profondeur (ainsi que celle du mouvement implicite) fournit le contexte formel. Or, lorsque la stratégie de la représentation devient, à travers l'apparition d'une réflexivité du cadre, «transitive ", l'image laisse apparaître un décalage inédit entre ce qui est donné à voir et ce qui est inféré par projection. Le cadre de la perception et le thème iconographique symboliquement interprété ne sont plus si strictement associés que l'un ne peut se percevoir sans l'autre. Comme dans ce mystérieux nuage que Mantegna a peint en forme de cavalier dans le Saint Sébastien de Vienne (fig. 9), quelque chose de l'ordre de la convention inconsciente qui régit tacitement la perception visuelle de l'espace se fait visible (Damisch 1972, Gamboni, ce volume : 147-156). Le processus de la projection se donne à voir. Exceptionnellement dissocié du cadre perceptif qui en occulte d'habitude l'existence, il laisse des traces sur l'image. L'enchâssement entre perception et projection, qui reste normalement à l'état de "sentiment inconscient de l'espace" (Hildebrand) montre ses limites, laissant entrevoir son caractère fictif.

Cette manière de mettre en évidence l'espace liminal apparaît assez tôt à la Renaissance, autour de 1530. Shearman a montré qu'à cette époque, Andrea Del Sarto, dans la Vierge aux harpies (fig. 10), insère dans sa composition des étranges «nuages d'encens» qui ne peuvent venir que de l'autel originellement situé sous le tableau. Très tôt remarquée et louée par Vasari (qui parle avec admiration d'une "fumée de nuages transparents sur l'architecture " surgissant derrière le groupe des figures [cité dans Shearman 1992 : 60]), cette invention confère sans doute " une étrange qualité d'atmosphère » (Shearman 1992 : 59) à la composition. Mais elle désigne aussi, indirectement, un espace liminal, qui marque la frontière entre ce que la composition donne à voir, et l'espace dans lequel cette même composition est située. L'apparition de la fumée révèle donc, pour ainsi dire par excès, le statut fictionnel de la peinture, et le degré, soigneusement calculé, d'illusion qu'elle génère. Véronèse va inventer, de ce même jeu, une version à la fois profane, élégante et vertigineuse dans les fresques qu'il a peintes à la villa Maser (fig. 5). Juan de Flandes en donnera, lui, une version dramatique et spectaculaire. À la fin de sa vie, vivant désormais en Espagne, il peindra une série de compositions, datant environ des années 1505-1506, où un saint Michel en armes, entièrement recouvert par une cuirasse en métal noir, écrase sous ses pieds un animal monstrueux (fig. 11). Sur la surface noire de l'armure, qui réfléchit la lumière, Juan peint de véritables visions d'Apocalypse, où des champs de batailles dévastés et des villes incendiées semblent surgir, comme dans un miroir sombre et miopaque, de l'espace où l'observateur est situé. Comme dans La Décollation de saint Jean-Baptiste exposée au musée d'Art et d'Histoire de Genève, le symbolisme est là, mais les conditions de son interprétation ont changé. Il ne s'agit plus ici seulement de déchiffrer un sens caché, mais aussi d'établir un rapport nouveau entre l'espace de la fiction et la place de l'observateur. L'invention de l'armure-miroir de saint Michel fait de l'observateur (jusque-là simple «lecteur » du sens symbolique) un protagoniste de la scène représentée : la ville incendiée et ses scènes de violences se trouvent, grâce au second foyer de la représentation que l'image en miroir situe à l'extérieur du tableau, derrière ses propres épaules. 
Fig. 11 Juan de Flandes, Triptyque de saint Michel, détail, vers 1506.

Musée diocésain, Salamanque @ Album /Oronoz/akg-images.

(3)

2. Les analyses que Gell (1996, 1998) a consacrées aux techniques qui, dans certains arts non occidentaux, tendent à piéger l'œil dans une représentation labyrinthique, sont sans doute à interpréter comme des cas de transitivité. Un autre exemple, sur lequel on ne peut insister ici est celui de l'art funéraire chinois, et notamment d'un certain nombre de monuments funéraires (datant de 618-713, dynastie T'ang), où, comme l'a montré récemment Jonathan Hay (2010), la représentation de la tombe est disposée du point de vue de l'esprit du mort.
Interprétation de la signification symbolique, projection du cadre, explicitation et exploration des actes de regard où perception et projection s'articulent. Des opérations esthétiques de ce type ne sont propres ni à la Renaissance, ni au maniérisme, ni à l'âge baroque. On pourrait même penser qu'un grand maître du $\mathrm{xx}^{\mathrm{e}}$ siècle comme Barnett Newman, dans la splendide série des Onements peints à New York dans les années 1950 (voir fig. 1, p. 4), explore, par les moyens de la peinture abstraite, précisément la trace de cette double présence de l'observateur. À travers un calibrage exact des dimensions de la toile et de l'intensité de la lumière, celui-ci se trouve en fait placé simultanément devant et au sein de l'espace, à la fois infini et aniconique, que l'image donne à voir. De Juan de Flandes à Andrea Del Sarto, de Bramantino à Mantegna, de Dürer à Barnett Newman, il est clair que les décalages entre thème iconographique et cadre, entre perception et projection ne sont ni épisodiques ni rares dans notre tradition.

Une image peut en cacher une autre, exposition consacrée à l'ambiguïté visuelle qui s'est tenue au Grand Palais en 2008, permet de faire un pas de plus. JeanHubert Martin et Dario Gamboni y ont montré que ces jeux réflexifs, qui mobilisent, chacun à leur manière, autant le symbolisme que le cadre de la représentation, ne sont propres ni à une époque, ni à une culture spécifique. La relation entre un cadre de la perception et son contenu, que nous avons rapidement mise en évidence en ce qui concerne la convention visuelle de la perspective (et qui, dans notre tradition, va constituer un des axes de la modernité, de Piranesi à Goya, de Manet à Newman, à Jasper Johns ou Markus Raetz), appartient sans doute aux termes constitutifs de toute représentation par l'image. Toute tradition iconographique possède sa propre transitivité, si on la définit comme la forme implicite d'illusion (ou d'appel au regard) qu'elle implique ${ }^{2}$. C'est donc du point de vue des formes de la relation entre projection et perception que nous pourrons maintenant revenir sur le concept de chimère, pour préciser selon quelles modalités cette relation entre réflexivité du cadre et espace liminal d'une part, et perception et projection d'autre part, peut s'établir dans le cas de la représentation chimérique.

Mais arrêtons-nous encore sur un point, qui concerne la distinction entre ambiguïté visuelle et représentation chimérique. L'image double ou potentielle, et plus généralement l'univers des décalages visuels qui peuvent s'établir entre la perception et la projection, ont été utilisés, dans la tradition primitiviste, comme une clé pour interpréter tout ce qui, dans les arts non occidentaux, mobilise l'ambiguïté visuelle. On a pu ainsi, dans cette perspective, comparer un nu de Degas qui se révèle être simultanément interprétable comme un paysage (fig. 24), à un cimier $A$-tshol de la tradition baga où plusieurs êtres sont représentés simultanément (fig. 15). On peut y voir, en effet, si on lit l'image de gauche à droite, la tête d'un homme, et, de droite à gauche, la forme d'un oiseau. En suivant ce même principe, ce masque pourrait à son tour renvoyer, en tant qu'être pluriel, à une enluminure moghole du xviI ${ }^{\mathrm{e}}$ siècle, où apparaissent des êtres féeriques composés de plusieurs animaux (voir fig. 6, p. 145). On passerait ensuite à une peinture d'Arcimboldo, et, de là, à Dalí ou à Johns. Dans tous ces cas, on verrait à l'œuvre le même esprit de "double signification ", ou de déchiffrement d'une image implicite. Il s'agit d'une erreur, qui dérive d'une réduction drastique, non pas des «significations » évidemment différentes de ces images, mais précisément de la stratégie d'invention visuelle qui, à chaque fois, y est à l'œuvre. Comme on va le voir, une image double, ou composite, n'est pas nécessairement chimérique. 


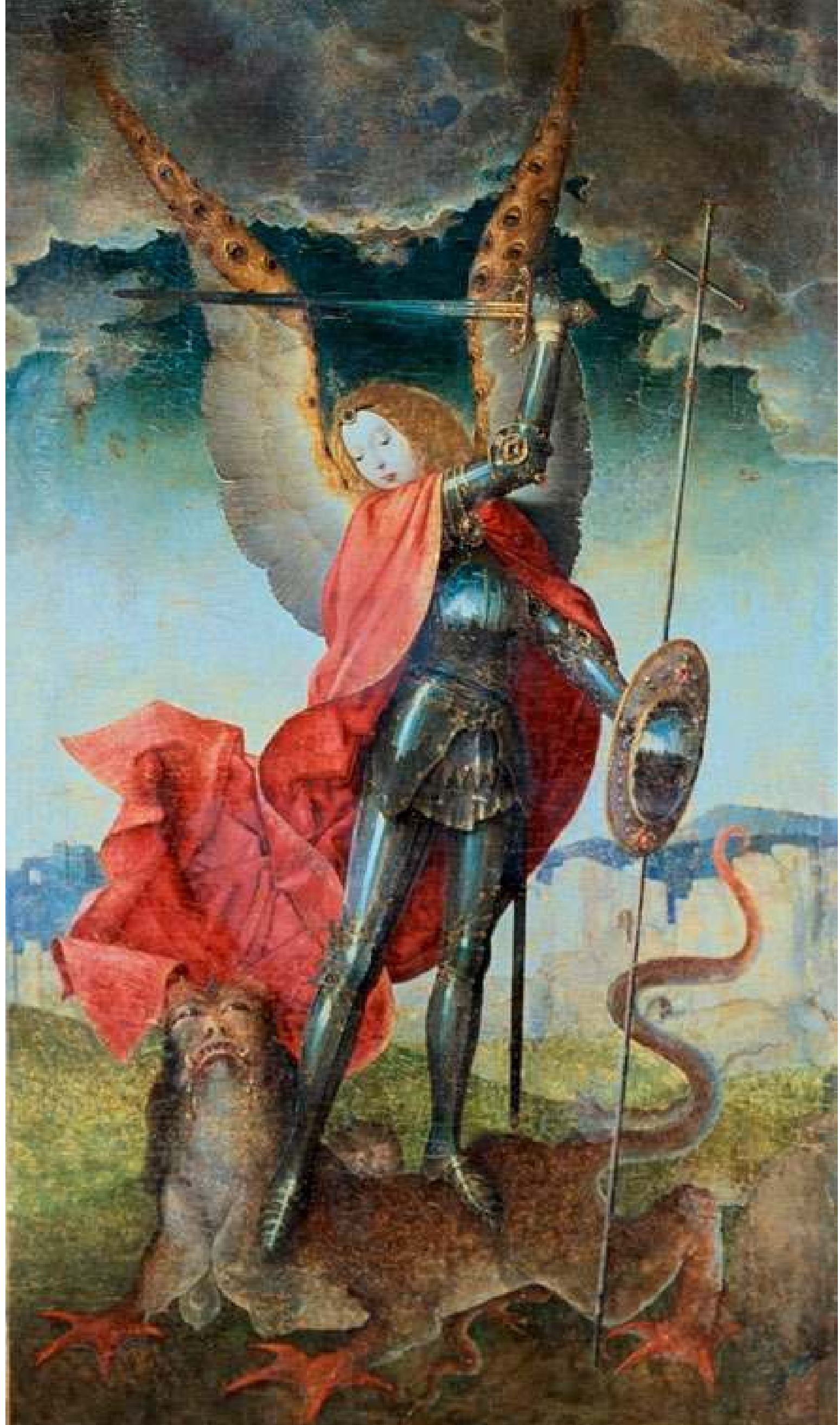


Considérons, une nouvelle fois, un exemple. Le musée Getty possède un heaume fort singulier, dit traditionnellement Heaume de Philippe $V$ de Macédoine et daté entre 350 et $300 \mathrm{av}$. J.-C. (fig. 13). Il s'agit bien, à première vue, d'une image multiple : sur le sommet du heaume surgit en effet la représentation d'un animal mythique, une sorte de griffon au bec d'oiseau. Ce détail a conduit les spécialistes à l'interpréter comme un objet rituel, probablement associé au culte que le roi Philippe vouait au héros Persée. Mais cet artefact nous intéresse aussi pour une autre raison. Une invention y apparait, bien plus intéressante de notre point de vue, et qui nous permettra de préciser notre langage et nos outils d'analyse aussi bien en ce qui concerne la morphologie de la représentation chimérique, que pour éclairer la logique iconique qui y est à l'œuvre. Tout autour du visage, dans cette zone du heaume que Riegl (2009) et Shearman (1992) nous ont appris à appeler liminale (et qui comprend ici, comme son extrême limite, le front et les sourcils), l'auteur de cet admirable artefact a gravé, très légèrement, avec une maîtrise technique extraordinaire, les contours des sourcils et les traces d'une chevelure. Ces cheveux savamment disposés sur le front et autour des oreilles, où ils rejoignent les poils de la barbe, marquent ici la frontière entre l'artefact et la personne dont la présence est imputée. Nous comprenons immédiatement, en effet, qu'ils appartiennent au jeune guerrier censé porter le heaume. Le premier effet de cette invention est de rendre perceptible (et donc pertinent) un espace vide. Si, par un acte de regard conscient, nous focalisons notre attention sur le contour de cheveux, qui se dispose tout autour du front et jusqu'au menton du guerrier, ce n'est pas l'image du célébrant qui apparaît dans l'espace vide qui devient subitement sensible autour de l'objet, mais bien un ensemble d'indices (réalisés avec une maîtrise extraordinaire) de sa présence imputée. Lorsque, au sein du regard, le heaume et le guerrier apparaissent ensemble, ce n'est nullement une image double qui surgit. Si nous avons recours au langage analytique de Peirce (1978), nous constatons que pour désigner un être pluriel, l'invention du sculpteur évoque d'une part, une icône, et d'autre part, un indice, sous forme de fragment visuel.

On a ici, par rapport aux exemples de représentation double ou composite que l'on a pu évoquer, deux transformations radicales. D'une part, on ne trouve jamais dans l'univers des représentations chimériques, dont le Heaume de Philippe V de Macédoine est un mémorable modèle, de redoublement d'image. Toujours, même dans les situations d'élaboration formelles les plus accomplies, la représentation plurielle est composée d'une image donnée à voir, et d'une autre qui est donnée à penser. D'autre part, le surgissement de la tête invisible du jeune guerrier, bien qu'implicite, n'apparaît pas en tant que décalage marginal par rapport à un modèle d'organisation de l'espace qui, comme la perspective, possède une existence indépendante. La pluralité est dans ce cas, comme dans tous les exemples de représentation chimérique qu'il nous a été donné d'étudier, le principe même de l'organisation de l'espace qui oriente, dans un même mouvement, et l'exercice de la perception et celui de la projection. Il ne s'agit pas dans ce cas d'un espace fictif, régi par des règles abstraites (par exemple, comme ici, géométriques), où une transgression apparaîtrait au niveau du symbolisme ou de la transitivité de l'image. Le lien entre le visible et l'invisible coïncide ici avec la définition même de l'espace : sans cette pluralité de regard, qui est d'emblée offerte, aucun espace liminal, entre ce qui est donné à voir et ce qui est inféré, ne surgirait. Le chemin de l'image chimérique est donc tout autre que celui de l'image double. 


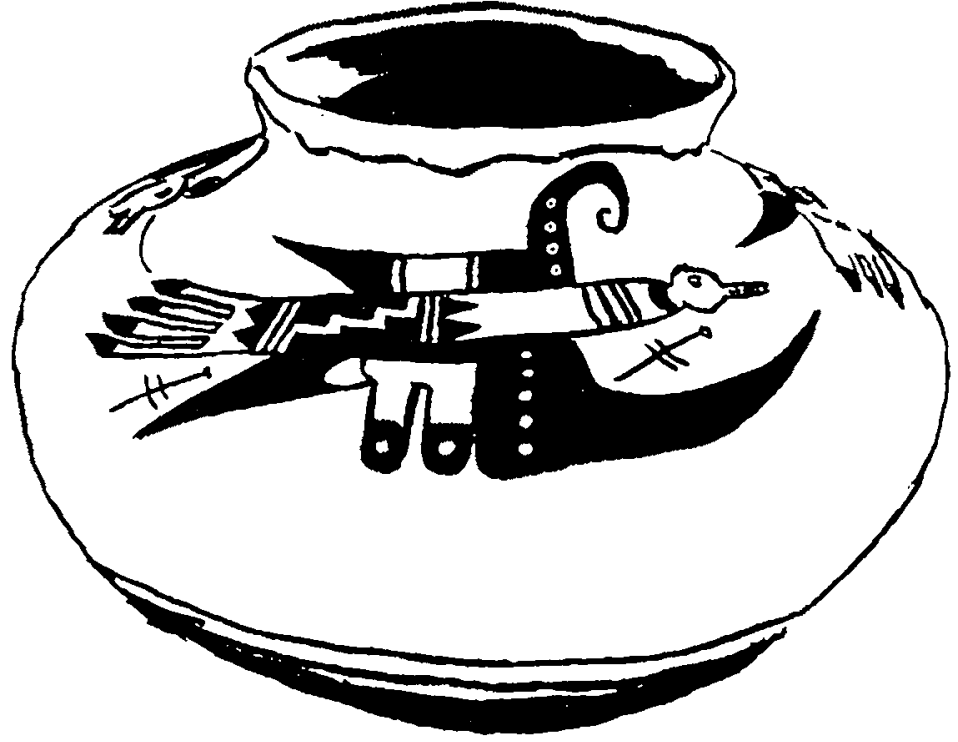

Reprenons le cimier baga (fig. 15) que nous avons cité. S'il y a bien, dans cette représentation, une élaboration par l'image d'une frontière entre ce qui est donné à voir et ce qui s'offre à la projection (tête humaine ou oiseau), aucun dédoublement de l'image n'y apparaît. Comme le heaume (dont il multiplie le principe, puisqu'on peut l'interpréter selon des directions différentes, de droite à gauche et vice versa), et comme la chimère hopi (fig. 12), que nous avons analysée ailleurs (Severi 2007), ce cimier n'est pas double. Les contours qui marquent ici la frontière entre perception et projection (les thèmes visuels "oiseau ", "nuage ", "foudre », etc.) ne fonctionnent pas comme des icônes qui feraient écho à d'autres images, mais comme des indices d'une présence à déchiffrer. On n'a donc pas ici, comme par exemple dans le paysage-femme de Degas (fig. 24), interférence, ou écho, entre deux représentations iconiques, à l'intérieur d'une même définition du cadre visuel. Là où on cherchait un redoublement, on trouve une articulation inédite entre l'image d'une totalité et celle d'un fragment, qui fonctionne non pas comme révélation d'un simple implicite, mais comme la préfiguration possible d'une présence entièrement différente, voire, le plus souvent, antagoniste. Ni sous forme potentielle, ni sous forme matériellement réalisée, on ne décèle ici la présence de deux icônes qui renverraient, par ambiguïté ou double sens, l'une à l'autre. Ce qui caractérise cette représentation, et la rend pleinement chimérique, est le renvoi, par une indication iconique fragmentaire, à une présence représentée par indices, qui ne se fait image que lorsque le regard, mobilisant "des capacités interprétatives, des catégories, des modèles et des habitudes de déduction et d'analogie» qui forment «ce qu'on peut appeler le style cognitif » d'un observateur donné (Baxandall $1985: 48$ ) fonctionne par projection.

La lecture du Heaume de Philippe $V$, ainsi que du cimier baga, nous permet donc de formuler deux premières indications du degré de complexité qui caractérise la représentation chimérique. L'une concerne le passage de l'ambiguité visuelle du statut de décalage entre projection et perception (dans le cadre d'un espace formulé par des moyens optiques indépendants) au statut de principe d'organisation de l'espace. L'autre concerne la mise en place d'une articulation logique entre une représentation iconique et une marque indiciaire de présence. On pourrait en conclure que si l'on veut comprendre les représentations chimériques, il ne sera pas suffisant de les assimiler hâtivement à un phénomène d'ambiguïté
Fig. 12 Oiseau-serpent hopi, céramique polychrome, style D. 


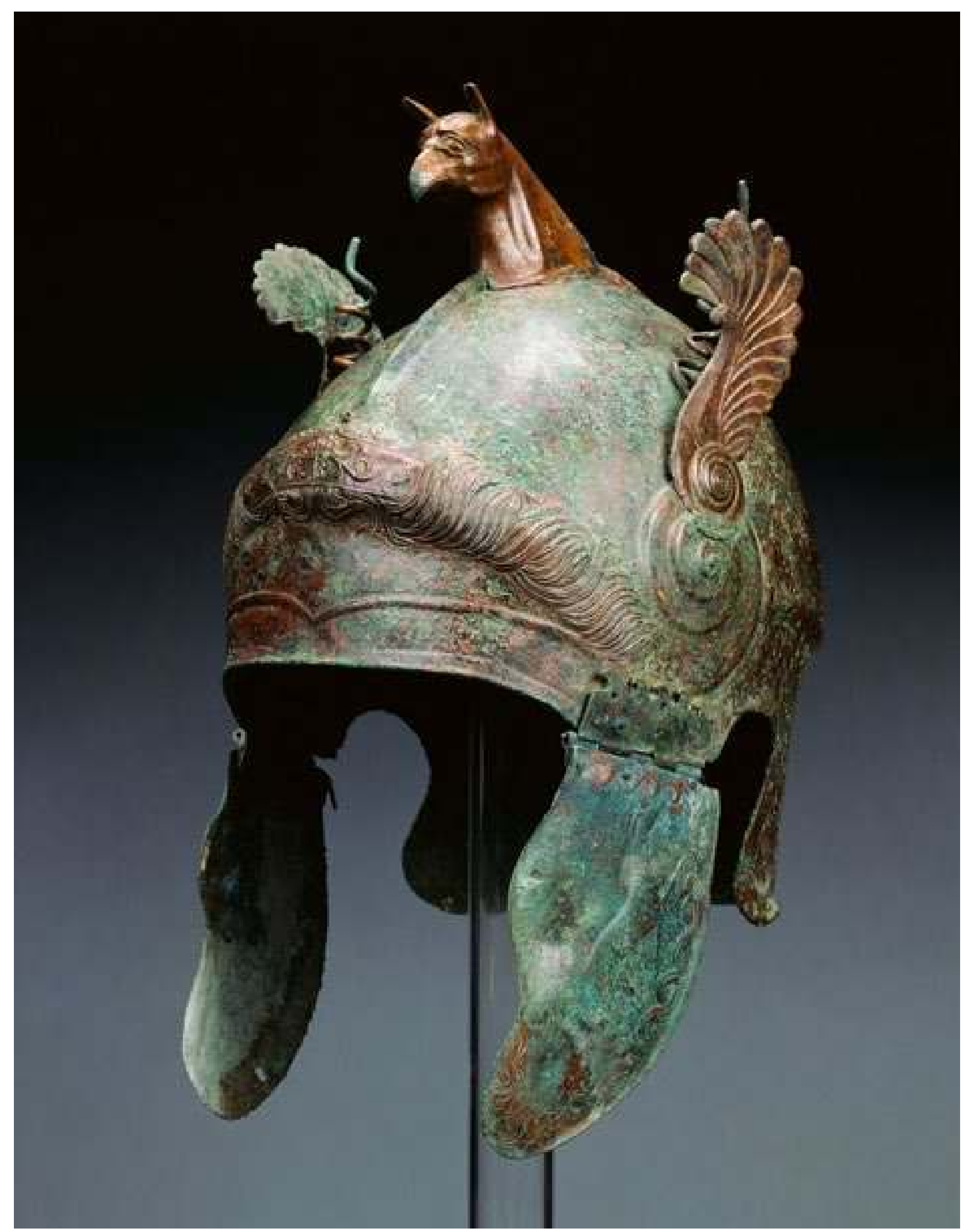

Fig. 13 Anonyme, Heaume de Philippe V de Macédoine, vve siècle av. J.-C., bronze $(28$ × 20 × $66 \mathrm{~cm}) \odot$ The J. Paul Getty Museum, Villa Collection, Malibu, California. 
visuelle. Il faudra, au contraire, apprécier avec exactitude le champ des possibles qui caractérise leur complexité propre. On assumera, comme point de départ, une définition qui comprenne, dans le travail de la pensée suscité par l'image, aussi bien les aspects purement optiques que l'ensemble des processus de déduction déclenchés par la représentation (et donc l'ensemble des phénomènes de projection qu'elle suscite). On dira alors qu'est chimérique toute image qui, désignant à travers une seule représentation un être pluriel, mobilise, par des moyens purement optiques ou par un ensemble d'inférences, ses parties invisibles.

À partir de cette première définition, on pourra répondre aux trois interrogations qui ont surgi à propos de la notion de chimère : une question morphologique (comment définir un type général de représentation chimérique? s'agit-il d'une représentation typique des arts non occidentaux?), une question logique (quel exercice de la pensée caractérise ce type de représentation?) et une question esthétique (qu'est-ce qui est propre à l'appréhension d'une représentation chimérique?).

Du point de vue morphologique, est propre à ce type de représentation un principe d'organisation de l'espace qui, tout en déclenchant plusieurs types de projection, fait du décalage entre une forme donnée à voir et une forme imputée le moyen d'engendrement d'une illusion spécifique (dans le sens que Gombrich [1969] et Florenski [1992] ont pu donner à ce terme). Dans cette perspective, à une typologie des iconographies (qui distinguerait entre images "réalistes", «abstraites» ou «symboliques») on préférera une typologie des espaces, définis comme l'ensemble des formes possibles de la relation, établie par la pure vision ou par l'inférence, entre forme et fond. On en conclura ainsi qu'une représentation fragmentaire mais non plurielle, comme le Tableau $n^{\circ} 1$ : Lozange avec quatre lignes et gris de Mondrian (fig. 14), ou plurielle mais non fragmentaire comme l'enluminure moghole (voir fig. 6, p. 145), ne sont ni l'une ni l'autre des représentations chimériques dans le sens que nous proposons de donner à ce terme.

Du point de vue logique, on réservera le terme «chimérique » à l'articulation spécifique entre représentation iconique (par imitation et convention) et indication indiciaire (visuelle, tactile, ou autre) d'une présence dont le mode d'existence est avant tout mental, et non matériellement réalisé. Il s'agit d'une image imputée par la pensée dont la réalisation n'est jamais considérée (précisément comme dans la boucle sibérienne du Pitt Rivers) que comme un indice. Cet indice peut apparaître sous la forme d'un fragment désignant la région liminale de l'image, comme dans le cas du Heaume de Philippe $V$, ou selon la mise en ordre d'une série de fragments, comme dans le cimier baga ou la chimère hopi.

Du point de vue esthétique, enfin, ce qui caractérise l'espace chimérique n'est ni une relation d'enchâssement stable entre perception et projection, comme dans la représentation symbolique dans le cadre de la perspective, ni un décalage épisodique où le cadre de la perception devient l'objet même de la projection. Il s'agit plutôt d'une relation instable, mais nullement confiée au hasard, de complémentarité alternée entre le thème iconographique et son espace liminal. Au sein de cet espace, un fragment visuel peut, certes, devenir intelligible grâce au fond dans lequel il apparait. Mais l'inverse est toujours possible. Au sein de cette convention visuelle spécifique, le fond pourra échanger de rôle avec la forme : c'est précisément ce qui se passe, en deux étapes, dans la double boucle sibérienne qui nous a servi de point de départ. C'est ainsi que, au sein d'un acte de regard qui vise à la désignation d'un être multiple, une image, dont le sens est acquis par projection, fonctionne comme principe (latent ou réalisé) de construction (ou cadre organisateur) de la perception de l'autre. 
$\mathrm{Au}$ sein d'une représentation chimérique, une réflexivité du cadre existe, parallèlement à un registre d'interprétation symbolique. Mais ce qui est donné comme cadre perceptuel (même sous la forme élémentaire d'un fond) peut toujours devenir principe d'interprétation projective (donc forme en tant que témoignage du travail du regard, au sens d'Hildebrand), et inversement. Loin de s'inscrire dans un cadre visuel fixe, la chimère reflète un jeu constant de présupposition réciproque entre perception et projection. Puisque, au sein de ce type d'espace, projection et perception ne peuvent s'exercer qu'en établissant une complémentarité provisoire, la forme et le fonds, le fragment et la totalité, le focalisé et le périphérique échangent sans cesse leurs rôles.

Bien que le déchiffrement des significations (comme on le verra dans les exemples amazoniens que nous allons étudier) y soit toujours présent, ce jeu de complémentarité possible entre thème et cadre n'investit pas, en premier lieu, le niveau du symbolisme. Si le fragment qui s'offre à l'interprétation projective a un sens (il désigne, par exemple, dans un système comme celui de la Côte Nord-Ouest, une nageoire, une tête, un bec d'oiseau, une queue; ou, en Amazonie, un jaguar, un vautour ou un anaconda), ce qui rend possible le jeu des renvois est son caractère fragmentaire, et donc sa relation au cadre qui engendre une forme spécifique de réflexivité, très loin de celle que nous avons identifiée dans le cas, plus familier, de la perspective. C'est ce jeu incessant entre fragment et cadre réflexif, qui rend l'espace chimérique, comme on va le voir dans les cas amazoniens, itératif, récursif, et potentiellement infini.

Reprenons, pour conclure ces premières réflexions sur la représentation chimérique, le masque haïda dont l'étude nous a permis, avec quelques autres exemples, de formuler ce concept. L'interprétation projective de certains traits de l'image («c'est un visage!») fournit dans un premier temps le cadre visuel pour désigner la présence d'un être humain, même si un détail, celui du nez, reste d'interprétation difficile. Mais il est toujours possible d'inverser le jeu : lorsque, en interprétant le nez du visage comme un bec, on dira «c'est un corbeau!», le reste du visage humain se pliera, par une sorte d'anamorphose spontanée, à représenter, autour de ce bec, la tête d'un oiseau. Encore une fois, le cadre rendra possible l'interprétation projective et inversement. Ce qui, dans le système occidental, n'apparaissait que comme un décalage épisodique ou exceptionnel, devient ici un principe d'organisation de l'espace, ce qu'on pourrait appeler une illusion sans perspective, qui n'est plus fondé sur la perception d'une profondeur, mais plutôt sur l'appréhension des limites (et des relations possibles qui peuvent en surgir) d'une image donnée. Ce principe peut se développer soit en termes de vision, de l'opposition duelle à la série, soit en investissant les relations qui peuvent s'établir entre image, son, parole, et représentations du mouvement. Précisons toutefois qu'aucun des traits que nous avons identifiés ne suffit à définir un type iconographique canonique de la représentation "chimérique ». Le travail qui nous a permis de les identifier conduit à une tout autre opération : il s'agit d'éclairer, au-delà de la chimère en tant que représentation, d'une part les coordonnées de l'espace chimérique, et d'autre part une logique des relations exprimées par l'image. Une chimère ne représente pas des êtres, mais des relations, possibles ou pensées comme telles, entre des êtres. L'idée de représentation chimérique ne s'inscrit pas dans une typologie des iconographies, mais bien dans une logique des relations iconiques, qui se déploie autant dans les images que dans les actes de regard qu'elles impliquent. 
Voyons maintenant un exemple de cette logique des relations entre les êtres que l'image chimérique permet de formuler en termes iconiques. Considérons deux traditions iconographiques amérindiennes où la représentation chimérique constitue sans doute la convention visuelle dominante : les Yekwana et les Wayana. Il s'agit de populations de chasseurs et agriculteurs tropicaux, qui parlent différentes langues de la famille caribe, et qui vivent aujourd'hui dans la région du Haut-Orénoque, entre Brésil et Venezuela. Le cas des vanneries des Yekwana, où toutes sortes de créatures mythologiques sont représentées, nous permettra d'introduire les premiers éléments de notre analyse. Les travaux d'un certain nombre d'ethnologues (Civrieux 1970, Wilbert 1981) nous ont permis d'acquérir une connaissance relativement détaillée de la mythologie de ces chasseurs et agriculteurs amazoniens. Il s'agit d'un long cycle d'histoires, relatant les épisodes sanglants d'un conflit qui, aux yeux des Indiens, régit tout l'univers. Ce conflit oppose Wanadi, personnage positif associé au soleil et présidant à la culture des humains (techniques d'agriculture, de pêche, de chasse, de fabrication d'artefacts, etc.), à son frère jumeau Odosha, qui incarne le mal, les malheurs, les maladies et la mort. Ce conflit cosmique ne représente pas, pour les Yekwana, un simple schéma d'explication de l'origine de l'univers. Remontant à l'origine des temps, la lutte entre ces deux frères ennemis n'a jamais cessé : elle marque la vie quotidienne des hommes, et entraîne souvent des conséquences tragiques. Cette rupture d'équilibre tient à une dissymétrie originelle entre le bien et le mal, et entre l'existence des humains et celle de leurs ennemis potentiels, animaux ou végétaux. Pour les Yekwana, le mal prévaut toujours sur le bien. C'est pour cela que Wanadi, leur allié, habite dans une région éloignée du ciel, et entretient peu de relations avec le monde d'ici-bas. Son jumeau Odosha, entouré de ses démons (souvent représentés par des «maîtres» invisibles des animaux et des plantes) est, lui, constamment présent, proche et menaçant. Ceci explique aussi qu'Odosha puisse être représenté par une longue série d'êtres maléfiques - singes hurleurs, serpents, jaguars, ou étrangers cannibales -, alors que Wanadi, réfugié dans son ciel, est seul à défendre les Indiens. En effet, chaque acte lié à la pêche, à la chasse ou à l'agriculture s'accomplit, pour les Yekwana, contre la volonté d'une foule de "maîtres invisibles" qui sont censés posséder les animaux et les plantes. Cet univers peuplé d'ennemis potentiels, toujours menaçant, est celui d'Odosha et de ses démons. Chaque acte nécessaire à la vie des humains suscite donc une vengeance qui, bien que constamment conjurée par des chants spécifiques, est toujours attendue. À ce principe de dissymétrie entre le bien et le mal s'ajoute l'idée d'un processus de transformation constant de l'un dans l'autre : toute acquisition culturelle (qu'il s'agisse d'armes, de vanneries, d'ornements ou de peintures corporelles) est pour les Yekwana le résultat d'une transformation du mal, ou des êtres qui en dépendent. D'où l'idée d'une constante ambiguïté qui frappe tous les êtres de l'univers : tout ce qui est utile et bénéfique (y compris les paniers en vannerie que les hommes décorent, en préparation de leur mariage) inclut une "part transformée » d'un être maléfique.

Guss (1989) a montré que la mémoire visuelle de la mythologie repose sur une iconographie spécifique, qui restitue une sorte de "catalogue» de ces êtres, et de leurs noms. En fait, au lieu de tenter de représenter tel ou tel épisode dans un espace plus ou moins "réaliste", les vanneries yekwana reflètent un niveau plus profond d'organisation du savoir mythologique: chaque être s'y trouve 
associé, par des moyens purement graphiques, avec sa part d'invisible. Comme nous l'avons vu, les deux grands motifs de cette mythologie sont l'opposition constitutive entre deux grands groupes de personnages et l'idée d'un processus de transformation continuelle les affectant tous. Ces métamorphoses ont deux modalités. D'une part, on peut avoir la notion d'une créature multiple qui (comme Odosha) "prend la forme » de toute une série d'autres êtres. On va donc, de ce point de vue, de l'individu à la série. D'autre part, ce processus de métamorphose incessante (où l'idée du bien résulte nécessairement d'un processus de domestication du mal) peut conduire à investir une même créature d'une ambiguité constitutive, qui en fait simultanément une instance positive et négative. On passe ainsi d'une série d'êtres à la représentation d'un seul être complexe. Or, l'iconographie yekwana permet de traduire en termes visuels, avec économie de moyens et précision, ces deux principes d'organisation du monde mythique. Les thèmes visuels qui traduisent les noms des esprits dérivent tous d'un même thème graphique, une sorte de " $\mathrm{T}$ » inversé qui représente Odosha. Grâce à quelques transformations géométriques simples, toute la série des autres personnages de la mythologie est engendrée à partir de ce premier thème graphique. Ces graphismes traduisent à la fois la multiplicité d'animaux différents (singe, serpent ou crapaud) et leur unité en tant que formes dérivées d'un même être originaire. Les différents personnages sont ainsi construits à partir d'une seule forme de base, dans un système qui permet de représenter non seulement des êtres bien identifiés, mais aussi leurs relations possibles. Ces relations entre figures (analogie, inclusion ou transformation) indiquent une organisation interne, propre à un système de représentations, qui se fonde évidemment sur un seul critère : il s'agit toujours de représenter, par la voie chimérique, la pluralité potentielle de chaque créature mythologique. Mais il y a plus. La technique visuelle que nous venons de décrire implique aussi un jeu de forme et de fond permettant de représenter (à travers une interprétation réflexive du cadre qui engendre, comme dans toute représentation chimérique, un jeu de complémentarité entre projection et perception) à la fois un être spécifique et une des ses métamorphoses possibles. Cette possibilité d'une représentation en forme d'être potentiellement double concerne plusieurs personnages de la mythologie : les singes, les chauves-souris ou les crapauds. L'exemple le plus frappant est sans doute celui du thème graphique dit woroto sakedi ("masque du jaguar », fig. 16) qui représente alternativement, selon qu'on focalise l'attention sur la forme ou sur le fond de l'image, Odosha ou Awidi, qui est une de ses transformations en forme de serpent. On reconnaît ici la relation instable, de complémentarité alternée, entre le thème iconographique et son espace liminal, et entre perception et travail de la projection, qui caractérise l'espace chimérique. En fait, comme l'a bien vu Guss, le vrai sujet des graphismes yekwana n'est pas tel ou tel personnage, mais « la relation dynamique en forme de transformation latente » de l'un dans l'autre (1989: 106,121-124). Nous trouvons donc, dans cette série iconographique apparemment simple, une organisation de l'espace proprement chimérique, qui se déploie par complexité croissante, à partir d'une forme élémentaire, partout présente et partout transformée. Au sein de cet espace, tout être (y compris Wanadi lui-même) résulte de la forme d'Odosha. Des ajouts, des variantes, des rapports d'inclusion, de répétition et d'inversion s'établissent entre ces formes, et en manifestent ainsi l'unité profonde. Par cette technique, qui joue à la fois sur le symbolisme et sur un type spécifique de réflexivité du cadre, l'univers chimérique de la mythologie se traduit en termes visuels. 


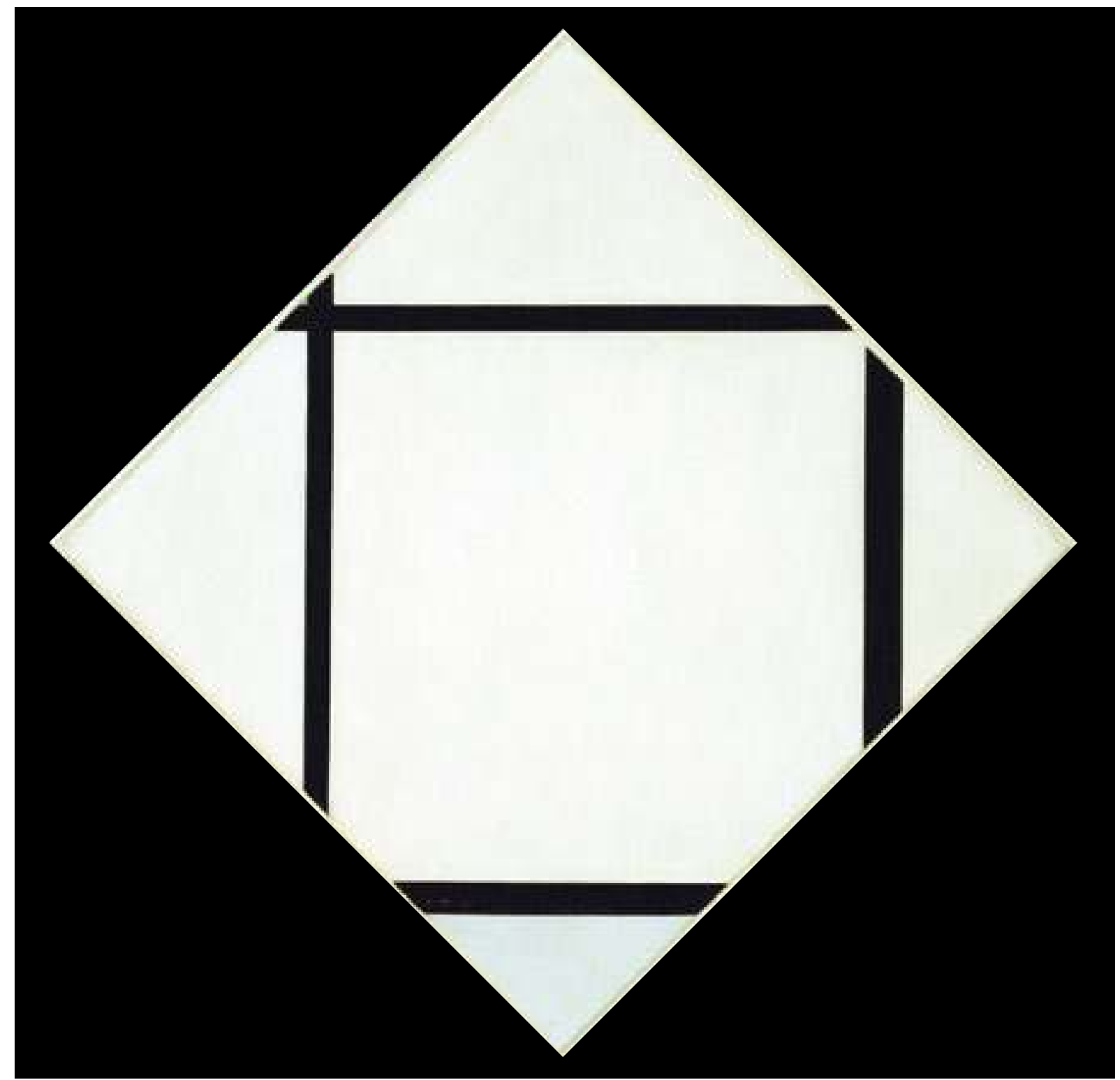

Fig. 14 Piet Mondrian, Tableau $n^{\circ} 1$ : Lozange avec quatre lignes et gris, 1926, huile sur toile $(113,7 \times 111,8 \mathrm{~cm})$. Museum of Modern Art, New York (c) 2011, Mondrian/Holtzman Trust c/o HCR International Virginia/Digital Image @ 2011, The Museum of Modern Art/Photo Scala, Florence. 


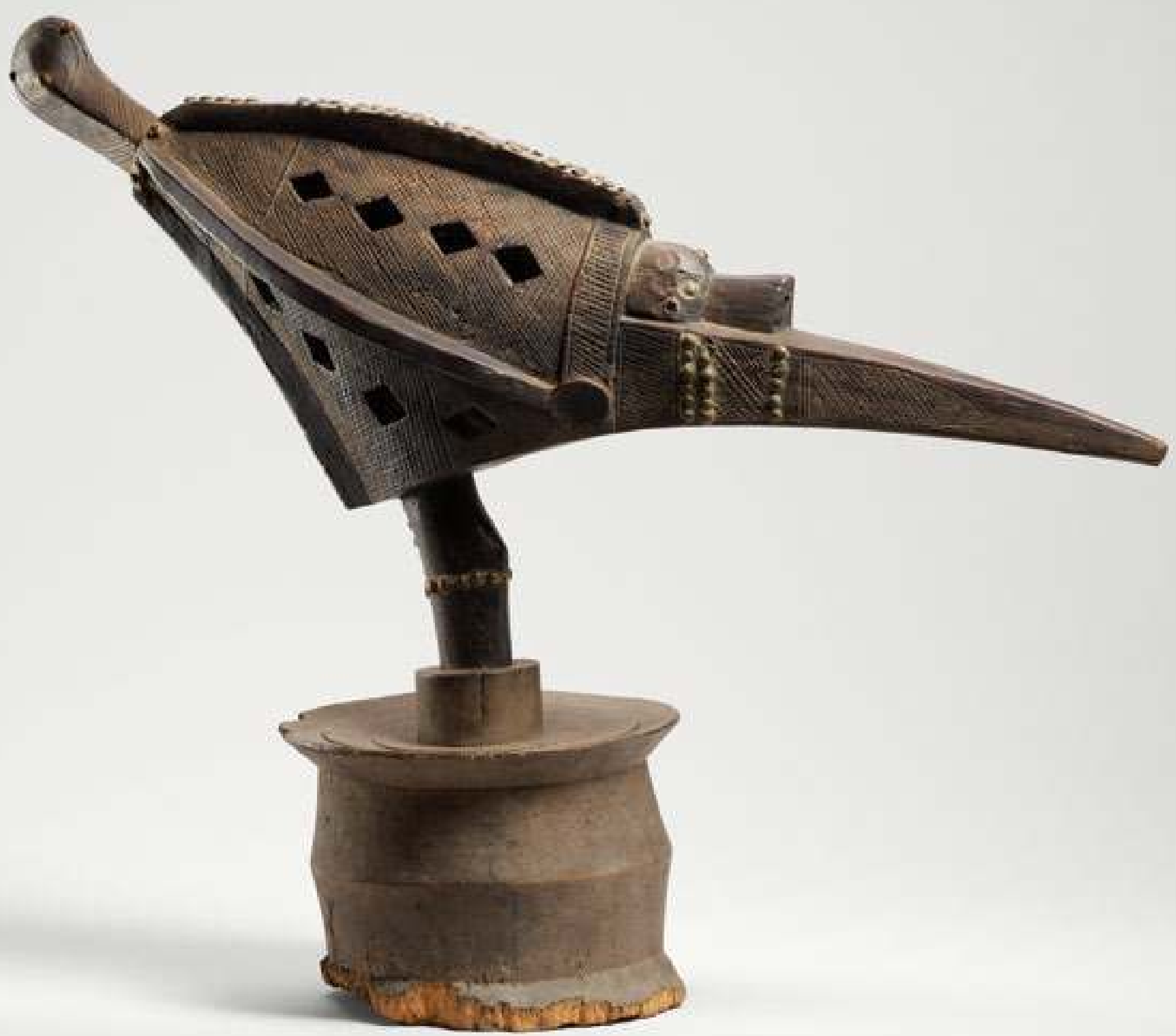


Ce type d'iconographie, où la représentation des êtres est indissociable de la représentation de leurs relations, est loin d'être exceptionnel ou isolé dans l'aire amazonienne. Une brève analyse des vanneries des Wayana, voisins des Yekwana dans la région du Haut-Orénoque, permet de montrer comment cette logique propre à l'image, fondée sur la notion de variation à partir d'un ensemble de modèles graphiques relativement élémentaires, peut se déployer pour atteindre une grande complexité. On trouve chez les Wayana une conception de la représentation iconographique (et même certains thèmes graphiques, comme celui qui est associé au jaguar [Velthem 2003 : 352-356]) très proche de celle des Yekwana. Pour eux, comme pour leurs voisins, une représentation iconographique s'organise toujours autour de motifs géométriques simples, comme le triangle, le carré, la spirale, les lignes croisées ou parallèles. Pour eux aussi, l'univers de ce type de représentation ne concerne que le récit mythologique, son commentaire et sa mémoire - bien que cet exercice de mémorisation des histoires traditionnelles semble moins formalisé chez les Wayana, où, selon Lúcia Hussak van Velthem, on trouve plutôt un schéma narratif général, concernant la prédation, qui offre néanmoins d'innombrables occasions de commenter en termes mythiques des expériences liées à la vie quotidienne. Mais l'ethnographie wayana se distingue de celle des populations voisines par la complexité du discours relatif à la représentation visuelle. Un thème géométrique n'est pas seulement, pour les Wayana, la marque ou l'emblème graphique d'un être mythologique. Il est le reflet d'une connaissance spécifique, nommée wayaman, qui se trouve métaphoriquement située dans la prunelle de celui ou de celle qui connaît la technique de la vannerie. Le wayaman est la "figure inversée " d'un esprit sous forme humaine qui se présente dans la prunelle de celui ou de celle qui fabrique un objet, et qui constitue le véritable « auteur » de l'objet. Ce type de connaissance (ou plutôt cette perspective, ce type de regard qui révèle la "vraie nature» des vanneries) concerne avant tout la forme des objets. Conçue comme une "pensée", mais aussi comme le reflet de cet « autre » qui habite les yeux de celui ou celle qui construit un artefact, et qui, pour ainsi dire, "guide sa main ", cette forme ne se révèle pleinement que lorsque l'objet est accompli selon les règles de la technique traditionnelle, ce qui permet à l'artefact de révéler sa vraie nature, et de se montrer «semblable à un être vivant ». En fait, selon la tradition wayana, les artefacts, les humains et les non-humains peuvent (et parfois doivent) partager la même décoration. C'est alors qu'ils "assument la même peau ". Cette notion est très importante, puisque, pour les Wayana, "la peau, ou plutôt la peau peinte selon un schéma reconnaissable, représente l'élément qui permet d'identifier la nature d'un être, le moyen par lequel on peut définir sa spécificité propre" (Velthem 2003: 129). C'est parce que les artefacts, et notamment les artefacts d'usage rituel, portent la même peau que les êtres ancestraux prédateurs, dont les modèles sont l'anaconda, le vautour ou le jaguar, qu'ils en sont toujours pensés comme les «répliques » ou l'«imitation ». Grâce à cette «identité de dessin », les artefacts peuvent donc "danser", "parler", ou même "attaquer " comme le font les prédateurs. En fait, les Wayana ne se limitent nullement à affirmer, comme les Yekwana, que les vanneries sont des «objets-corps». Puisque leur créateur a fabriqué la première femme humaine en utilisant précisément de la vannerie, un seul et même processus «engendre " à leurs yeux, même en termes sexuels, les artefacts et les humains. On dira non seulement que les vanneries, comme d'autres êtres 

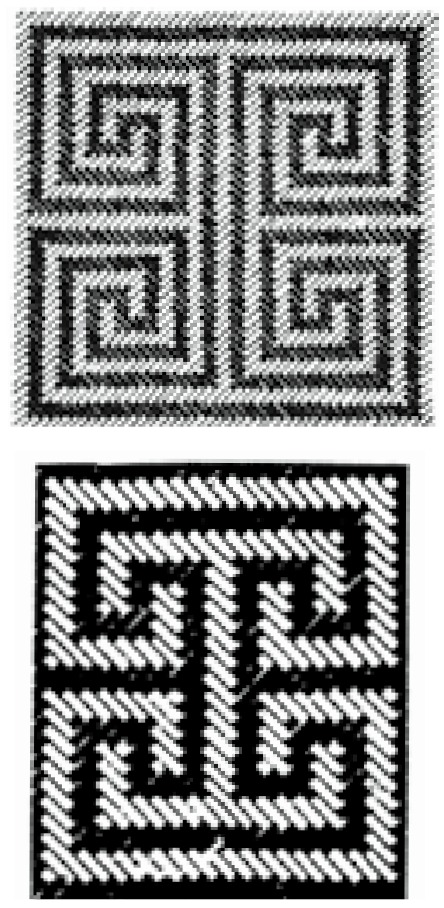

Fig. 16 Le thème « masque du jaguar " (woroto saketi), vannerie yekwana. vivants, sont douées de parole, de mouvement (ibid. : 197) ou d'un sexe (ibid. : 135), mais aussi que les humains et les animaux, précisément parce qu'ils peuvent porter les mêmes graphismes sur leur peau, sont composés de la même matière que les artefacts. L'idée de "peau peinte - explique Lúcia Hussak van Velthem - est indissociable de l'idée de copie, et de celle de reproduction, puisque c'est à travers cet élément que, selon les Wayana, tout être est engendré. La production de tout individu suppose la production d'une nouvelle peau, un acte technique qui se fonde sur l'observation d'un modèle préexistant» (ibid. : 240). Dans cette perspective, la peau d'un nouveau-né est «symboliquement associée à un tissu de plumes ». Celle d'un adulte est toujours pensée comme un tressage de dessins, une vannerie décorée. Bref, dans l'univers des Wayana, l'identification entre humains et artefacts ne se fonde pas sur une ressemblance directe, mais plutôt sur l'idée que tout être vivant est défini par une décoration ou un dessin spécifique, représentant à la fois sa peau, son emblème et son nom visuel.

Toutefois, nous aurions tort de penser que l'apparence des êtres du monde est fixée, pour les Wayana, selon des modèles préétablis. Puisque tout ce qui existe est pris, dans le monde des Wayana, dans un processus de transformation constante, tout être peut assumer, à chaque instant, la "peau » d'un autre, et même, parfois, de plusieurs êtres à la fois. Velthem rappelle le cas des danses qui ont lieu dans la maison des hommes. Celle-ci est censée être un lieu «habités par les poissons tukuxi ", qui sont d'ailleurs représentés, avec bien d'autres êtres, dans la roue centrale du plafond de la grande hutte cérémonielle. Mais les poissons sont aussi, à leur tour, représentés comme des "colibris au long bec ». Lorsque les hommes masqués «agissent en poissons", ils deviennent, en même temps, des « colibris au long bec ».

Cette idée de transformation potentielle et incessante de tous les êtres est très répandue dans l'univers amazonien. Chez les Yekwana, elle s'exprimait à travers l'opposition de deux frères ennemis, Wanadi et Odosha. Les Wayana partagent cette idée d'une dualité originaire des êtres. Pour eux aussi, les êtres du monde se divisent en prédateurs et non prédateurs. C'est même là une des premières tâches accomplies par leur créateur mythique, qui a, selon eux, littéralement bâti l'univers en distinguant les prédateurs des autres, aussi bien chez les animaux, les végétaux et les humains. Mais il ne s'agit nullement ici, comme chez les Yekwana, d'êtres individuels, doués d'une personnalité distincte. Là où les Yekwana inventent des personnages paradigmatiques, les Wayana raisonnent par classes. Au lieu d'opposer un Wanadi à un Odosha, ils distinguent différents modes d'existence qui peuvent caractériser n'importe quel individu, quelle que soit sa nature - animale, végétale, humaine ou artefactuelle. Profondément enracinée dans la pensée traditionnelle, cette catégorisation est aussi lexicalisée dans la langue. Prenons l'exemple de l'anaconda, qui constitue un des modèles du prédateur. «Ses actes de prédation - écrit encore Velthem - possèdent un caractère si paradigmatique que non seulement ils désignent toujours, en général, la dimension surnaturelle, mais ils peuvent aussi, en tant que tels, s'appliquer à tout autre espèce animale. [...] Cette conception permet d'attribuer à d'autres êtres, comme les larves de papillons, les mille pieds, les poissons et les oiseaux, des instincts de prédation dans un cadre surnaturel, associé à l'anaconda.» (ibid. : 105) Inversement, l'anaconda va, à cette occasion, " porter le nom et la peau » de ces animaux. Velthem remarque que : "L'identification de ce couplage d'êtres est signalée par les suffixes okoin ou koimë, qui signifient "en-tant-qu'anaconda", et s'appliquent au nom d'une espèce spécifique. » (ibid.) On aura par exemple, sous l'appellation 
de kiapo-koimë, le «toucan-en-tant-qu'anaconda ", représenté comme un serpent pourvu d'un long bec et dont la peau est couverte de plumes de couleurs contrastées. De la même manière, on prêtera à un oiseau fourmilier considéré « en-tantqu'anaconda", l'image d'un reptile au chant d'oiseau. Un processus analogue concerne le jaguar, dont la présence est signalée par un autre suffixe (kaikuxin), qui va engendrer des êtres qui, comme le rongeur quatipuru, peuvent être considérés et nommés "en-tant-que-jaguar».

Nous avons ici l'exemple de ce que nous serions tentés d'appeler des «chimères verbales » désignant des êtres multiples et changeants, que seule leur catégorisation à partir d'un suffixe commun définit en tant que membres d'une seule classe. Le concept de série, qui est aussi présent dans la tradition iconographique des Yekwana, apparait ici sous une forme beaucoup plus complexe. L'exemple le plus clair de cet engendrement de séries est offert par les danses d'initiation masculine analysées par Velthem, où les masques portés par l'initiant désignent un être multiple qui n'est pas seulement constitué d'esprits différents (arara, faucon, poisson, soleil, arc-en-ciel), mais aussi de formes multiples de ces esprits «en-tant-que » incarnations de différents prédateurs : jaguars, vautours ou anacondas (ibid. : 212). On passe ainsi de la chimère à la série d'êtres chimériques. À travers cette double série de marquages du corps de l'initiant, le concept de représentation chimérique atteint un niveau de complexité jusque-là inconnu. Le rituel devient un lieu de transformation où les jeunes garçons masqués vont progressivement "porter la peau peinte " de toute une série d'esprits animaux, végétaux ou humains, eux-mêmes sujets à d'innombrables métamorphoses. Le passage logique essentiel qui caractérise le cas wayana est donc bien celui qui conduit de la représentation d'individus différents (des personnages, avons-nous dit) à la représentation de membres de classes, et même, comme dans le cas de la danse rituelle, à la représentation de ce qu'on pourrait appeler des séries de séries d'êtres chimériques³.

Comment cette logique complexe, et l'ontologie qu'elle implique, se traduisentelles en termes visuels? Faut-il penser que le cas wayana n'a plus rien en commun avec celui, plus simple, des Yekwana, qui semblait se limiter à la traduction visuelle d'une série limitée de noms propres? Ou bien devrons-nous admettre que ces catégories de complexité croissante, que nous avons vues à l'œuvre dans l'action rituelle, n'appartiennent qu'à la dimension de l'exégèse, ne sont que de faits de discours, sans rapports avec l'iconographie?

La représentation d'êtres individuels sous forme de motifs géométriques simples, telle que nous l'avons rencontrée chez les Yekwana, n'est nullement absente de la tradition wayana. Velthem mentionne quarante-sept thèmes graphiques pour les vanneries et vingt-neuf pour les céramiques. Toutefois, les Wayana ne se contentent pas, comme les Yekwana, de simples listes de thèmes. Ils font jouer un principe de classification des thèmes graphiques regroupés en trois catégories distinctes : ceux qui "appartiennent" aux peintures corporelles de l'anaconda, ceux qui sont associés à la peau du jaguar et ceux qui se réfèrent à la peau des «monstres anthropomorphes» (une catégorie comprenant notamment les ennemis, dont les Blancs). Certains motifs visuels, qui gardent leur signification spécifique, sont ensuite associés à la désignation de groupes ou de catégories d'êtres. Considérons un premier cas. "Une des formes paradigmatiques de la prédation est l'acte de "blesser, piquer, perforer"." (Velthem 2003: 327) L'acte qui le résume, "flécher ", ou "atteindre en traversant la peau " est caractéristique d'un artefact, la flèche, et de plusieurs animaux, les cobras, les guêpes, les scorpions,
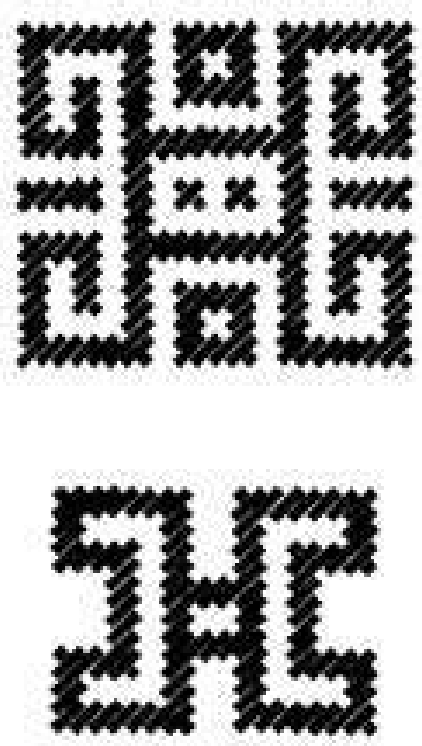

Fig. 17 Le thème complexe "crabe/œil de tapir", vannerie wayana.

- $\odot$

3. Les êtres considérés «en-tantqu'anacondas » seraient ceux capables d'étreindre et de dévorer les humains. Ceux qu'on associe aux «larves de papillons », ellesmêmes considérées «en-tantque-jaguars », comprendraient en revanche tous les êtres capables de «mordre de l'intérieur », et de manière presque imperceptible, les humains, par exemple à travers les maladies (Velthem 2003 : 320). 
et certains oiseaux, dont la cigogne maguari (Florida caerulea). "Cet oiseau, qui est reconnu comme le prototype des êtres qui piquent, est représenté au sein de l'iconographie Wayana par le motif dit "bec de maguari" [fig. 18], dont le contour graphique indique une position de vigilance, qui est le propre de cet animal. [...] En fait, ce graphisme représente aussi bien la flèche en tant qu'artefact que tout animal qui, considéré en tant que prédateur, est censé "atteindre" sa proie à la manière d'une flèche. La flèche redoublée pourra donc désigner, de manière assez indéterminée, "tout ce qui pique". On a ainsi une première manière de sortir de la représentation d'un être individuel pour passer à celle de la série. » Une fois établi, ce principe s'applique à d'autres cas. "Un thème graphique wayana, écrit encore Velthem, peut être multiple, et se référer simultanément à plusieurs êtres. " (ibid. : 313) L'image se décompose alors en plusieurs parties, qui possèdent chacune une signification indépendante. C'est ainsi que le thème graphique du «crabe» (fig. 17) contient aussi celui qui désigne l'« œil de tapir». L'interprétation s'effectue donc, dans ces cas, en suivant ce que Velthem appelle le «dialogue interne " des formes à l'intérieur des thèmes graphiques. Cette représentation d'un être à travers les thèmes graphiques qui désignent ses transformations possibles peut se réaliser aussi à travers le dessin d'un seul animal qui «porte sur sa peau » des thèmes graphiques représentant d'autres êtres. C'est le cas du jaguarcaramujo (colimaçon) (fig. 19).

Ces représentations d'un seul "être complexe" trouvent un écho saisissant dans les documents récoltés par Barcelos Neto (2002) chez les Wauja du HautXingu, où l'on retrouve des représentations d'anacondas surnaturels définis par des séquences de thèmes graphiques se référant chacun à d'autres animaux (fig. 20). Mais le système wayana est encore plus complexe. Nous avons, jusqu'à présent, étudié des cas dans lesquels une seule représentation iconographique se réfère à plusieurs êtres du monde. L'iconographie wayana connaît aussi le cas inverse, où des séries de dessins vont désigner collectivement un seul être. On retrouve là la notion de prédateur ancestral, un être qui, se manifestant à travers une série de métamorphoses, se trouve représenté comme un être collectif ou sériel. L'illustration la plus éloquente de ce processus est fournie par les peintures qui décorent, comme nous l'avons signalé, les «roues de toit» (maruana) de la maison cérémonielle (fig. 21). On y voit apparaître une série de thèmes graphiques qui renvoient à plusieurs êtres, eux-mêmes hybrides (les anacondas-crocodiles et des poissons qui "possèdent des caractéristiques propres aux mammifères et aux oiseaux"), mais dont la série représente, dans son ensemble, la « raie-entant-qu'anaconda ». Nous sommes en présence d'une sorte d'usage récursif du même principe de catégorisation. L'être chimérique wayana ne se contente pas d'associer plusieurs fragments d'êtres différents en un seul corps : grâce au principe d'autonomie des dessins par rapport aux différentes surfaces où ils peuvent apparaître, il peut non seulement associer des thèmes graphiques différents en un seul corps, mais aussi associer des thèmes différents en des corps différents, eux-mêmes combinés en des séquences qui représentent collectivement, comme ici sur la maruana, des êtres surnaturels conçus comme des séries de transformations (la raie-en-tant-qu'anaconda).

Remarquons aussi que, même lorsqu'elle réalise, sous plusieurs formes, le passage de la désignation d'êtres spécifiques à la représentation de séries de statut logique différent, l'iconographie wayana reste bien liée à la représentation de listes de noms propres, question dont nous avons souligné l'importance dans d'autres contextes (Severi 2007). Seulement, ces noms propres désignent dans ce 


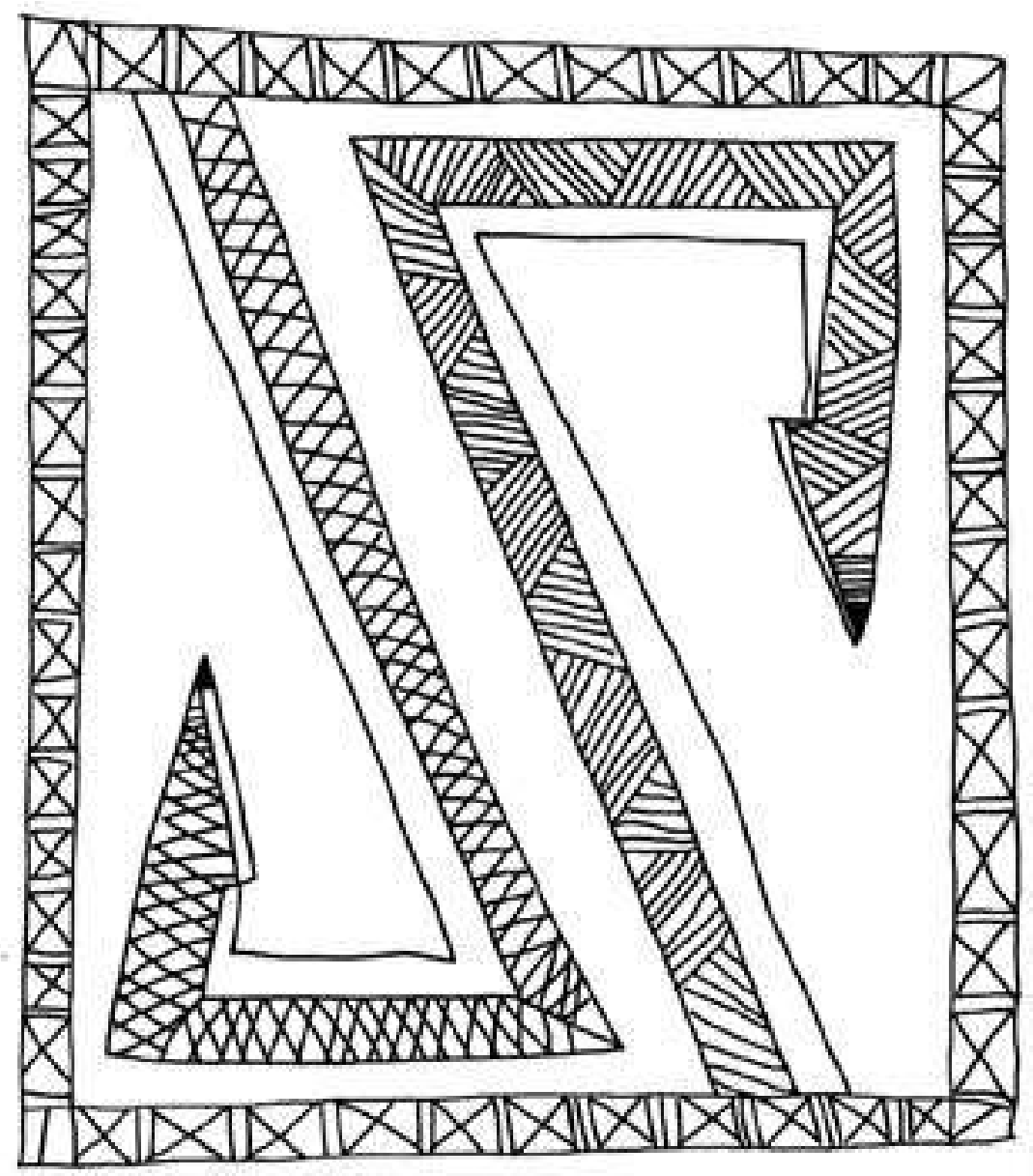

Fig. 18 Le thème cigogne maguari, vannerie wayana.

contexte non plus des personnages individuels (même "déguisés " ou transformés, comme pouvaient l'être, chez les Yekwana, Odosha ou Wanadi), mais des séries organisées d'êtres. Les noms traduits en termes visuels par des thèmes graphiques fonctionnent en fait chez les Wayana comme des « définitions verbales » qui mobilisent plusieurs noms d'espèces. On assiste donc à la mise en place, du point de vue de la mémorisation des noms, d'un double processus. Soit un seul thème graphique représente le nom visuel d'une série d'êtres, réunis pour l'occasion dans une seule classe, par l'utilisation de critères taxonomiques distincts. Soit une série de thèmes graphiques, disposés en séquences ordonnées, illustre la série de transformations qui désignent la "vraie nature " d'un seul être.

Il reste un troisième cas de figure, où l'être ancestral n'est pas représenté directement, mais se trouve désigné seulement par la relation, exprimée en termes exclusivement visuels, qu'il peut entretenir avec d'autres êtres. C'est le cas, par exemple, du rongeur quatipuru, qui peut être représenté, sans qu'il soit à proprement parler figuré, à travers sa relation avec d'autres animaux. On y verra à l'œuvre une autre manière de traduire en termes visuels le concept de « chimère verbale ", typique de l'esthétique de la prédation wayana, où plusieurs types de "passage à la série " se trouvent utilisés. Suivons la description donnée par Velthem, qui permet en ce cas de s'approcher encore de la conception wayana de l'iconisme. Sans doute guidée par ses interlocuteurs, Velthem distingue ici d'une 
Fig. 19 Le thème jaguar-colimaçon, peinture wayana.

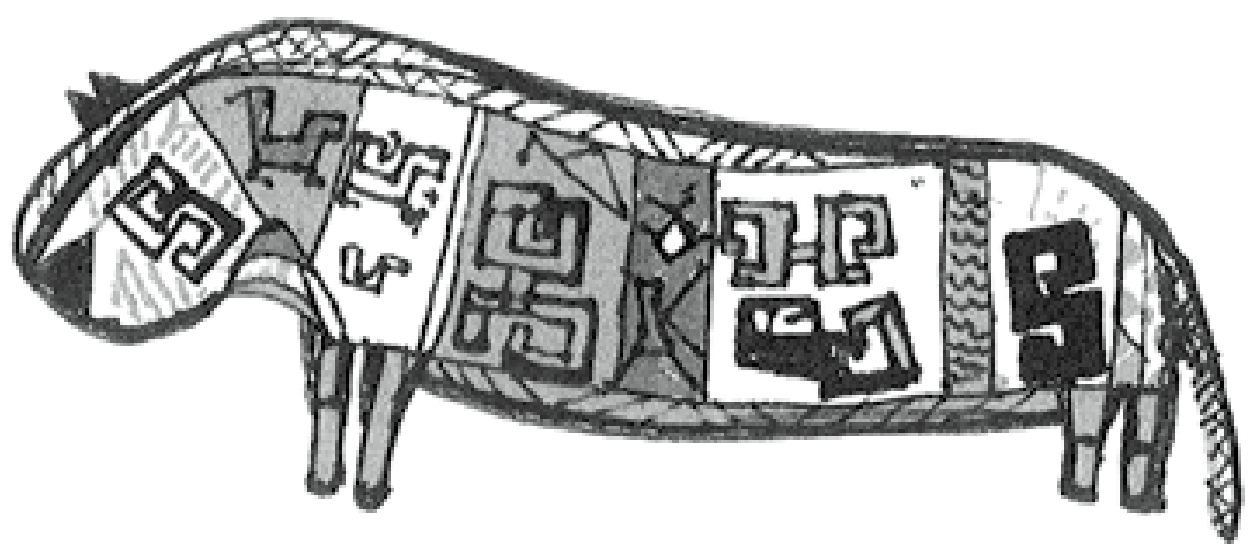

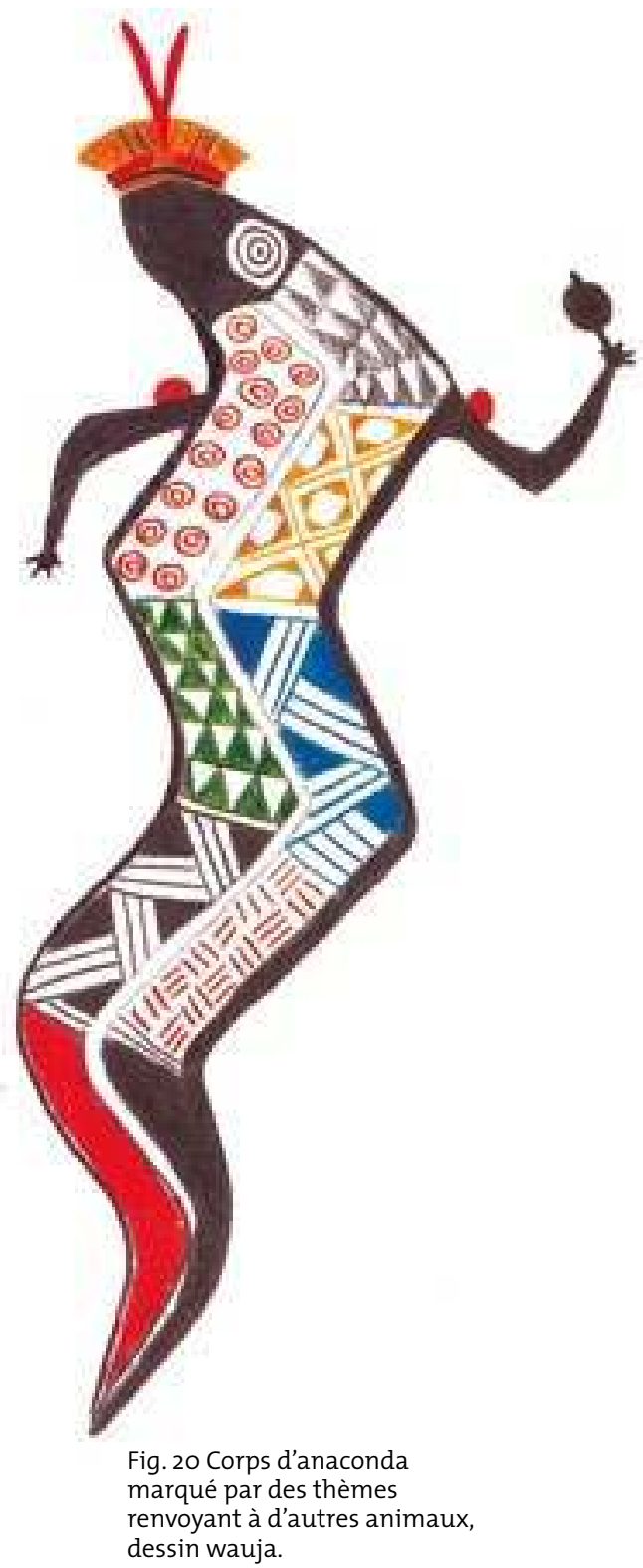

part l'ukuktop, l'«image perceptuelle » de l'animal tel qu'on peut le voir dans la forêt proche du village, avec sa morphologie, son comportement typique, la nourriture qu'il préfère, et d'autre part le mirikut, le thème graphique qui désigne ce même animal dans l'iconographie traditionnelle des vanneries (fig. $22 \mathrm{a}$ et $22 \mathrm{~b}$ ). "Si tout mirikut est naturellement une image, selon la distinction formulée explicitement par les Wayana, toute image est loin d'avoir le statut de mirikut", remarque Velthem (2003 : 317). Celui-ci permet en effet d'interpréter la vrai nature (ou de "déchiffrer la peau peinte») de l'animal dont on a quotidiennement l'image sous les yeux. Voyons comment cette opération constante d'exégèse des apparences peut se réaliser. Prenons l'exemple du quatipuru et du maguari, un petit rongeur et une variété de cigogne qui font partie du milieu familier des Wayana. Ils sont représentés dans l'iconographie traditionnelle par deux thèmes graphiques : la double flèche que nous connaissons déjà (fig. 18), et une figure géométrique, qui peut faire penser à une représentation « réaliste» du quatipuru (fig. 22b).

L'interprétation indigène de ces images procède de l'interprétation d'un thème isolé à celle de groupes de thèmes. Une fois reconnue la ressemblance des thèmes (mirikut) avec les «images perceptuelles " (ukuktop) de ces deux animaux, on affirmera d'abord que le mirikut diffère de l'image des animaux parce qu'il représente leurs « doubles surnaturels ». Le thème géométrique ne représente pas (seulement) l'un ou l'autre animal (familier et somme toute inoffensif) mais sa « réplique monstrueuse, et normalement invisible" (2003: 319). On se souviendra sans doute que la tradition wayana distingue entre différentes classes de thèmes, qui appartiennent à différents prédateurs. Lorsqu'on considérera de ce point de vue les deux thèmes, on reconnaîtra que la double flèche et le petit rongeur nommé quatipuru "appartiennent tous les deux" aux peintures corporelles qui figurent sur le ventre de l'anaconda, et c'est donc à ce prédateur qu'ils seront associés. Ils pourront même, en tant que membres d'une série, faire partie de sa représentation. Le thème du quatipuru se traduirait donc en ce cas, comme l'écrit Velthem, comme «image (ukuktup) et peinture corporelle (imirikut) de l'anaconda " (ibid. : 317). Toutefois, en tant que représentation d'un être invisible, le thème du quatipuru contient aussi une référence possible à un autre prédateur, le jaguar. 
Pourquoi? Il suffit de l'associer au thème graphique du jaguar (fig. 23) pour s'apercevoir qu'un seul détail les sépare : la queue, tournée vers l'extérieur dans le cas du quatupuru et tournée vers l'intérieur dans le cas du jaguar (ibid. : 318). Cette comparaison révèle donc un "point de connexion » inattendu entre les deux thèmes graphiques qui restait invisible lorsqu'il s'agissait d'associer le rongeur quatipuru à la cigogne maguari. Ce point en commun ne concerne naturellement pas seulement les graphismes, il concerne aussi les deux êtres représentés. On doit donc en conclure que ces deux animaux (lorsqu'on les considère du point de vue que leur mirikut révèle) possèdent tous les deux "un corps de félin ", ce qui confirme la nature de jaguar (ou plutôt le mode d'existence possible «en-tantque-jaguar ") du quatipuru. Nous reconnaissons ici le caractère essentiellement sériel de l'iconographie wayana : un être n'y est jamais saisi seulement dans sa singularité. Toujours, il est défini par «la peau peinte " qu'il porte, en tant que membre d'une classe, ou d'une séquence de "modes d'existences " possibles.

À propos de ce type de représentations, Velthem a parfaitement raison de parler de "formes mnémoniques» (2003: 319). Comme nous avons pu le constater dans d'autres cas (Severi 2007, 2009), les relations mnémoniques ne s'établissent nullement, comme les relations sémiotiques, entre un signe (ou un dessin) et son référent. Il s'agit plutôt d'un ensemble d'inférences visuelles, fondées sur le déchiffrement d'images complexes, qui établissent une relation entre d'une part une mémoire spatiale, qui concerne ici les thèmes graphiques, et d'autre part

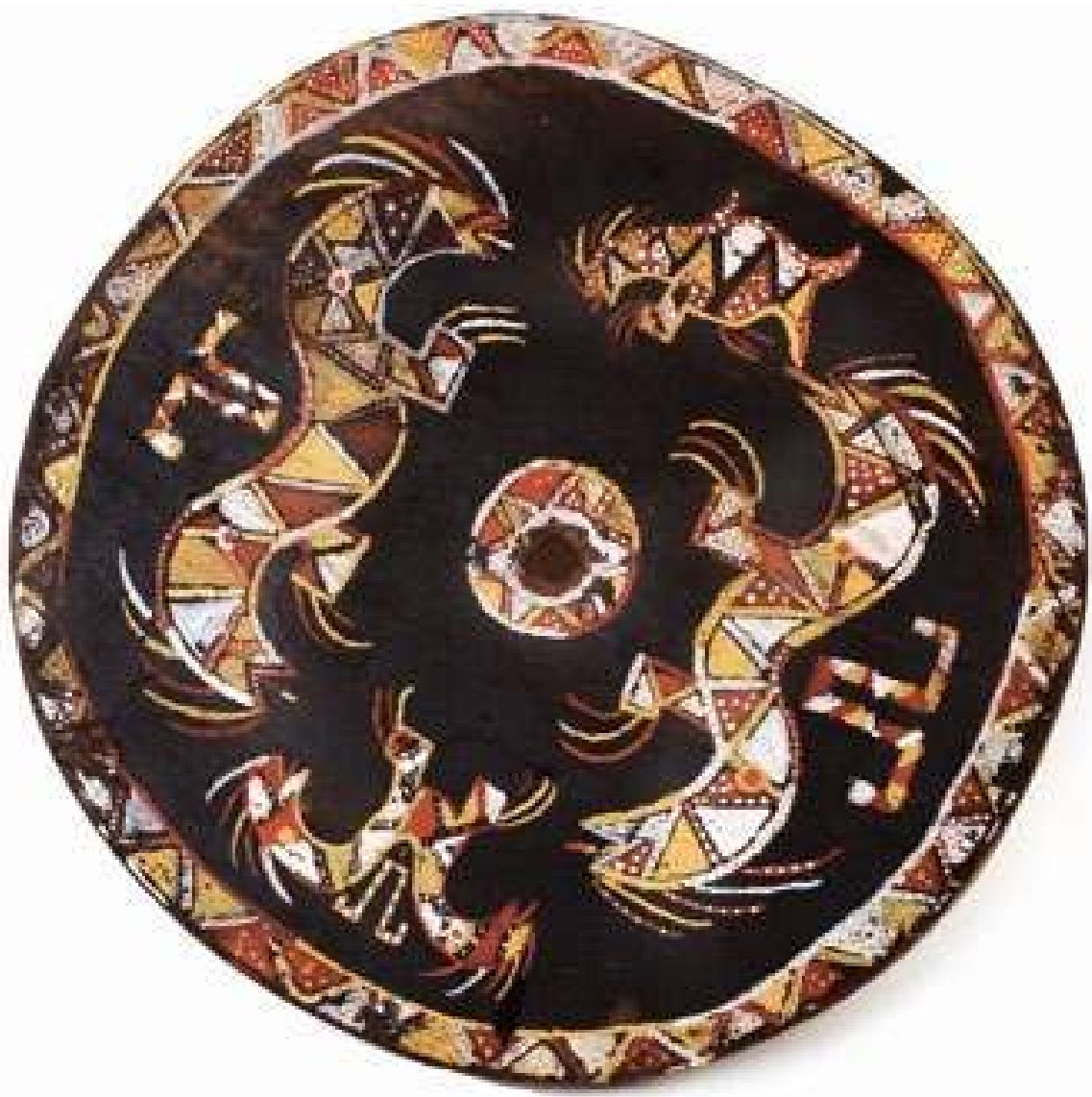

Fig. 21 Ornement plafonnier (manuara), xxe siècle, État de Para, Rio Paru de l'Est, Brésil, Wayana-Aparai () musée d'Ethnographie de Genève (MEG). Photo Johnathan Watts. 

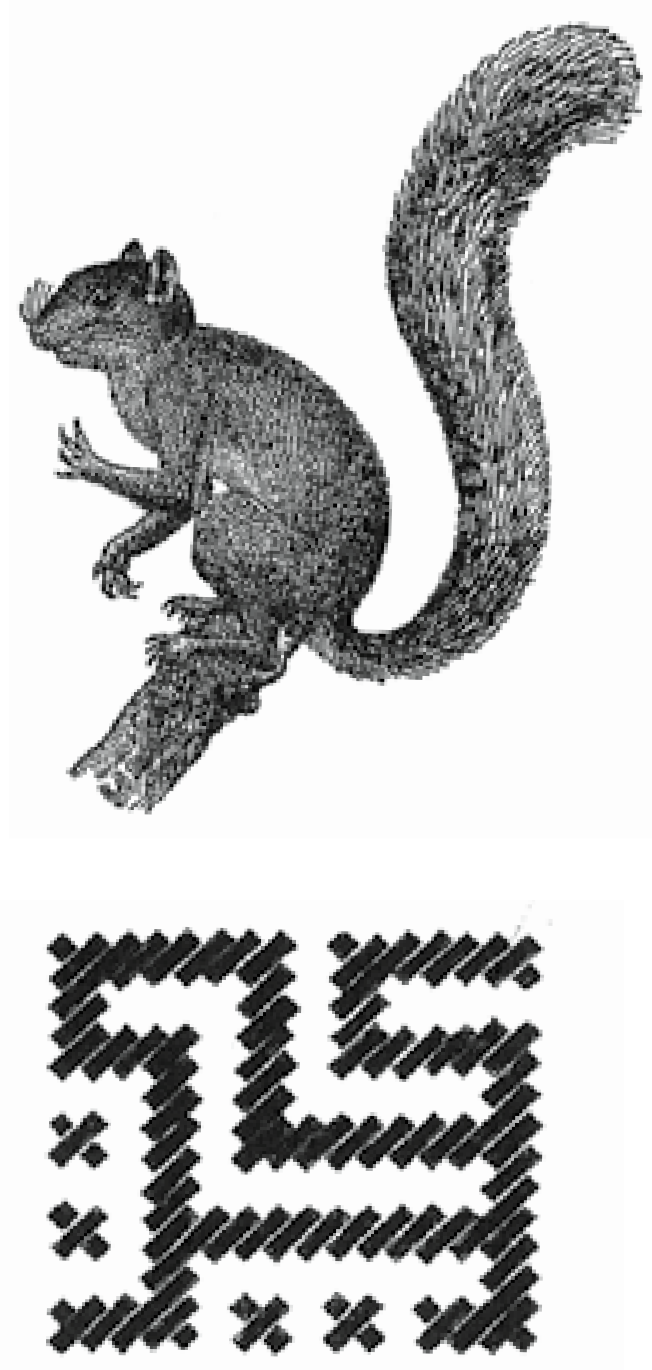

Fig. 22a Le quatipuru.

Fig. $22 \mathrm{~b}$ Le thème quatipuru, vannerie wayana. la mémoire des mots. L'efficacité des pratiques liées à la mémorisation des traditions iconographiques n'est donc pas due à la tentative plus ou moins réussie d'imiter la voie de la référence propre à l'écriture, mais à la relation que ces pratiques établissent entre différents niveaux d'élaboration mnémonique. Les cas yekwana et wayana désignent, de ce point de vue, deux différents degrés de complexité d'un même espace chimérique. Il est clair que l'on retrouve, dans le déchiffrement des thèmes graphiques wayana, les deux principes, d'ordre et de saillance, qui gouvernent la mémorisation des noms propres chez les Yekwana. L'établissement d'un ordre, dans ce nouveau contexte, affecte les séries iconographiques, liées aux «séries d'êtres» organisés par catégories de prédateurs. La mise en place d'une saillance propre à chaque thème permet, quant à elle, l'identification de chaque terme de ces séries, et de chaque série à l'intérieur de ce que nous avons appelé des «séries de séries ». Dans les deux cas, la pratique du déchiffrement des images chimériques suppose un exercice, orienté par la tradition, de l'inférence et de la projection. La confrontation entre les thèmes du quatipuru et du jaguar, qui se déploie simultanément sur le plan de la prégnance (ensemble du corps «félin» en commun) et celui de la saillance (queue à l'orientation opposée, vers l'extérieur ou vers l'intérieur), en offre un exemple très clair. Le graphisme wayana (mirikut) est donc une sorte de nom visuel, qui utilise des traits iconiques relevant de l'image naturelle (ukuktop) de plusieurs êtres pour construire, à travers leur mise en séries, l'image d'êtres complexes, que l'on dirait purement conçus, puisqu'ils échappent à la vue, s'il n'étaient pas, eux aussi, pris dans un processus d'incarnation constante et toujours provisoire.

Cette attention portée aux aspects potentiels et implicites de la représentation par thèmes graphiques, qui peuvent constamment se fragmenter et se démultiplier, dans une sorte de mouvement perpétuel de la représentation vers sa dimension chimérique, suggère que la logique de ces iconographies peut encore se développer. Il serait par exemple possible de transférer ces procédés de référence sérielle des images à des moyens d'expression autres que la vision. Une telle hypothèse de travail nous conduirait alors à étudier les relations qui s'établissent, notamment chez les Wayapi, qui appartiennent au même groupe linguistique et vivent dans la même région que les Wayana, entre des thèmes graphiques et des "images sonores" qui assument, par exemple dans les suites musicales appelées touré (Beaudet 1998), la fonction de représenter les prédateurs et leurs métamorphoses, attribuée ici aux seuls graphismes. On passerait ainsi des séquences, organisées selon un ordre géométrique simple, des Yekwana, aux représentations sérielles des Wayana, pour atteindre ensuite un niveau de complexité ultérieur, où les séries de représentations seraient composées aussi bien d'images visuelles que d'images sonores. On pourrait ensuite changer encore de moyen d'expression, et envisager l'usage que certains chants 
chamaniques font de l'onomatopée verbale, qui y apparaît comme une véritable image sonore des êtres, tout en restant lexicalisée, en tant que signe linguistique, dans la langue indigène. Il s'agit, en somme, d'un champ d'investigation très vaste, dont nous ne pouvons esquisser ici que quelques éléments, et qu'il faudra sans doute explorer encore.

Concluons pour l'instant que les traditions iconographiques que nous avons brièvement étudiées ici, yekwana et wayana, ont recours à des moyens comparables, soit en ce qui concerne la représentation géométrique de séries de traits (que l'on peut constamment réduire à leurs composantes élémentaires, ou organiser en configuration plus complexes), soit en ce qui concerne la relation, elle aussi variable, entre iconographie et langage. Dans les deux cas, la représentation du monde des esprits conserve toujours son caractère sémiotiquement hybride, "en même temps représentatif et conceptuel " (Velthem 2003: 306), puisque son iconisme, bien que raffiné et souvent imprévisible, est toujours associé à un nom propre, ou à une définition verbale (ce qu'on pourrait appeler un nom-série) de l'être représenté. Au sein de ce dernier système, dont l'univers reste - rappelonsle - limité au récit mythologique, un double rapport va s'établir entre la représentation iconographique et la définition verbale. Dans certains cas, on représentera par un seul thème graphique des séries d'êtres, ou des "noms propres complexes ", comme ceux de certains prédateurs. Dans d'autres cas, on utilisera des séries de thèmes graphiques désignant un seul être, grâce à son évocation comme "nom d'une série ", avec la raie-en-tant-qu'anaconda, ou comme terme intermédiaire implicite, avec le quatipuru-en-tant-que-jaguar, désigné par la juxtaposition partielle de son thème graphique et de celui du jaguar.

Chez les Yekwana comme chez les Wayana, l'espace chimérique est là, avec le jeu constant de complémentarité instable entre perception et projection qui le caractérise. La différence entre les deux iconographies concerne d'une part le passage de la représentation d'individus "personnalisés » à celle de séries d'êtres en transformation constante, et d'autre part le nombre de termes intermédiaires, de caractère linguistique ou iconographique, qui se trouvent mobilisés pour désigner un être spécifique. Là où l'ethnographie pouvait faire penser à des traditions différentes, l'analyse des thèmes iconographiques, et des opérations mentales qu'ils impliquent, révèle une unité sous-jacente. Une même logique, liée à la représentation chimérique de relations, est à l'œuvre dans les deux cas.

VIII

Pour répondre aux débats suscités par la notion de représentation chimérique, nous avons, dans un premier temps, essayé de formuler des outils d'analyse nouveaux dans le domaine de l'anthropologie des images. À partir d'une brève étude de la convention visuelle qui nous est plus familière, la perspective, nous avons ainsi défini l'appréhension d'une image comme une relation, variable et spécifique à une tradition, entre un cadre perceptif et l'exercice de la projection des savoirs acquis, ou des "catégories interprétatives " (Baxandall $1985: 48$ ) qu'il implique. Cette analyse nous a conduits à focaliser notre étude sur des notions potentiellement universelles, comme l'interprétation du symbolisme par projection, la transitivité des images, la réflexivité du cadre, et les actes de regard. Nous avons ensuite appliqué ces notions à la représentation chimérique, qui nous est apparue, du point de vue morphologique, comme fondée sur un principe d'organisation de l'espace qui fait de la relation entre une forme donnée à voir et une

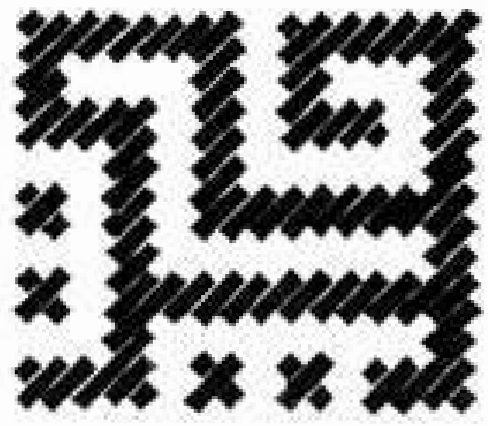

Fig. 23 Le thème jaguar, vannerie wayana. 
forme imputée par la pensée le moyen d'engendrement d'une illusion spécifique. Du point de vue logique, nous avons ensuite pu conclure que le type d'opérations mentales que cette représentation suppose se fonde sur une articulation spécifique entre représentation iconique et indication indiciaire. Du point de vue esthétique, enfin, nous avons pu conclure que l'espace chimérique désigne une relation instable, de complémentarité alternée, d'une part entre le thème iconographique et son espace liminal, et d'autre part entre la perception et le travail de la projection.

Ce travail d'analyse nous a permis de conclure que la représentation chimérique est avant tout une représentation de relations exprimées par l'image. De ce point de vue, elle ne s'inscrit pas dans une typologie des représentations iconographiques ("réaliste», «aniconique», «abstraite», etc.), mais bien dans une logique des relations iconiques. L'analyse de deux traditions amazoniennes, où la représentation chimérique constitue la convention visuelle dominante, nous a permis de montrer comment la désignation par projection d'une présence imputée engendre l'idée d'une essence propre aux êtres chimériques. Dans le cas yekwana, cette essence est représentée par des grands personnages, conçus comme des êtres soumis à une transformation constante, qui dominent les récits mythologiques. Dans le cas wayana, cette essence du chimérique est développée en une logique plurielle, où l'on voit apparaître, à la place de personnages, des classes (et parfois des classes de classes) d'êtres hybrides. Dans ces traditions, comme ailleurs en Amazonie, la représentation chimérique s'associe à la définition essentialiste d'une classe d'existants dont les traits définitoires ne coïncident jamais avec ceux qui, au sein du monde humain, animal ou végétal, définissent une espèce. Le jeu incessant entre fragment et cadre, et entre perception et projection, qui rend l'espace chimérique itératif, récursif, et - parmi les Wayana - potentiellement infini, désigne donc non seulement une esthétique, mais aussi un mode d'existence des êtres surnaturels dans le monde amazonien.

On pourrait en conclure que l'étude de la représentation chimérique permet d'éclairer d'un regard nouveau non seulement les arts, mais aussi l'ontologie de ces traditions. Tel est sans doute l'un des enjeux d'une anthropologie, dont nous avons ici esquissé les premiers éléments, de l'espace chimérique.

EHESS-CNRS, Laboratoire d'anthropologie sociale severi@ehess.fr 


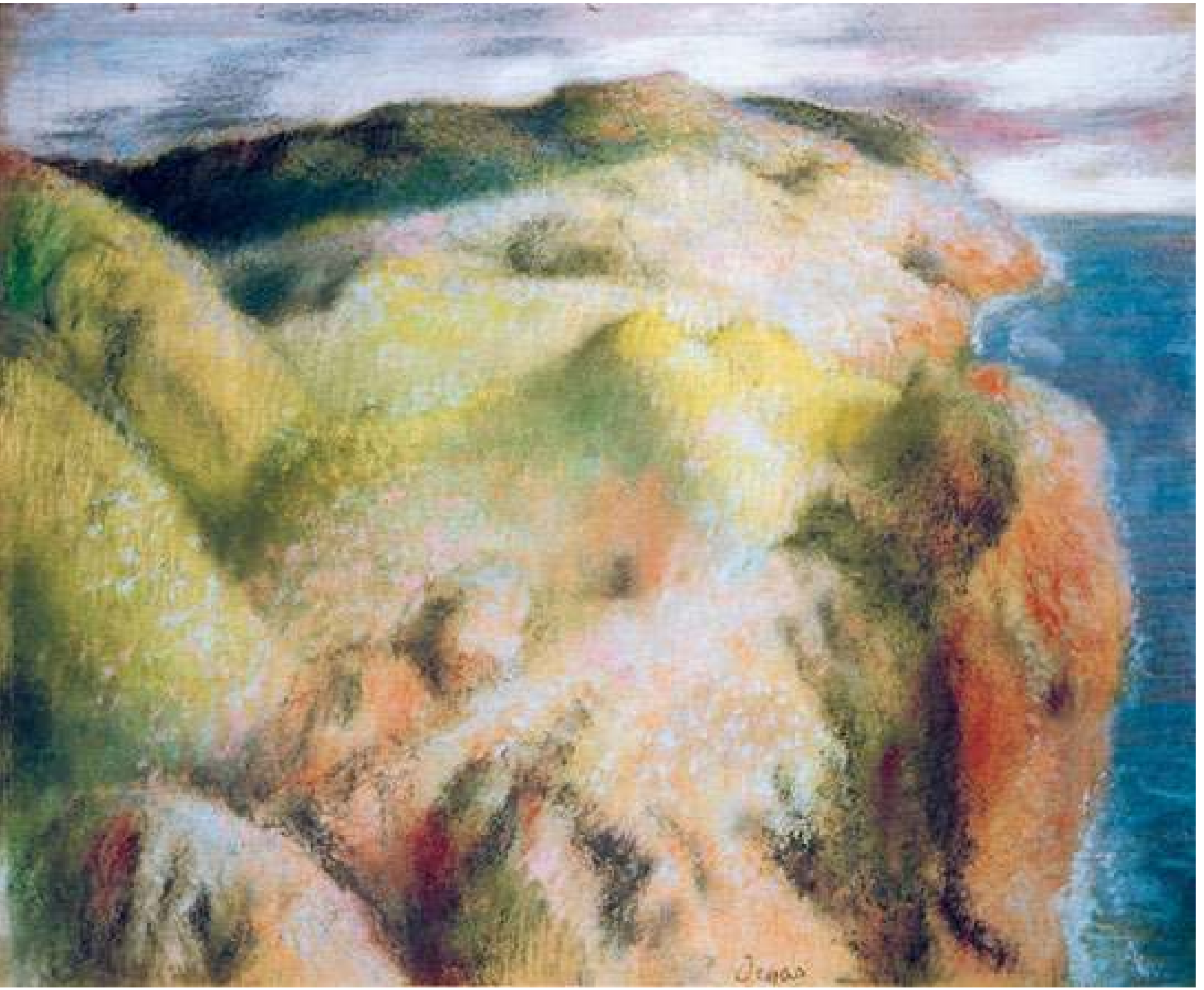

Fig. 24 Edgar Degas, Falaises, vers 1892, pastel sur papier $(42 \times 55 \mathrm{~cm})$. Collection particulière $\odot$ akg-images. 


\section{Bibliographie}

\section{Alpers, Svetlana}

2005 Les Vexations de l'art. Vélasquez et les autres. Paris, Gallimard.

\section{BARAsch, Moshe}

2003 «Renaissance Hieroglyphics 》, in Aleida Assmann et Jan Assmann (éd.), Hieroglyphen. Munich, Fink Verlag : 165-191.

Barcelos Neto, Aristóteles

2002 A Arte dos Sonhos. Lisbonne, Museu Nacional de Etnologia.

BASchet, Jérôme

2008 L'lconographie médiévale.

Paris, Gallimard.

BaxAndall, Michael

1985 [1972] L'EFil du Quattrocento.

Paris, Gallimard.

1989 [1986] Les humanistes à la découverte de la composition en peinture, 1350-1450, Paris, Gallimard.

\section{Beaudet, Jean-Michel}

1998 Souffles d'Amazonie. Paris, Société d'ethnologie.

BÜHLER, Karl

1990 [1934] Theory of Language: the Representational Function of Language. Amsterdam, John Benjamins.

\section{Civrieux, Marc de}

1970 Watunna. Mitología Makiritare. Caracas, Monte Ávila Editores.

DAMisch, Hubert

1972 Théorie du nuage. Paris, Seuil.

Diderot, Denis

1951 Euvres. Paris, Gallimard

[«Bibliothèque de la Pléiade »].

FLORENSKY, Paul

1992 La Perspective inversée. Paris, L'Âge d'Homme.

FrIEd, Michael

1990 La Place du spectateur. Esthétique et origines de l'esthétique moderne. Paris, Gallimard.

\section{GAMBOnI, Dario}

2004 Potential Images. Londres, Reaktion Books.

\section{Geli, Alfred}

1996 «Vogel's Net: traps as artworks and artworks as traps », Journal of Material Culture $1(1): 15-38$.

1998 Art and Agency. An Anthropological Theory. Oxford, Clarendon Press.

\section{Goethe, Johann Wolfgang von} 2000 [1808] Traité des couleurs.

Paris, Triades.

\section{GoMBRICH, Ernst}

1969 Art and Illusion. A Study in the Psychology of Pictorial Representation. Oxford, Phaidon Press.

1982 The Image and the Eye. Further Studies in the Psychology of Pictorial Representation. Oxford, Phaidon Press.

\section{Guss, David}

1989 To Weave and Sing. BerkeleyLos Angeles, California University Press.

\section{HAY, Jonathan}

2010 « Seeing through dead eyes. How early Tang tombs staged the afterlife », Res. Anthropology and Aesthetics 57-58 : 16-54.

\section{Hildebrand, Adolf von}

2001 [1893] II problema della forma e altri scritti. Palerme, Aesthetica Edizioni.

\section{LAMP, Frederick}

1996 Art of the Baga. New York-Munich, Museum for African Arts-Prestel.

\section{LÉvi-Strauss, Claude}

2008, Euvres. Paris, Gallimard

[«Bibliothèque de la Pléiade »].

\section{MARc, Franz}

1996 [1914] Les Cent Aphorismes.

Paris, Fourbis.

\section{PANOFsky, Erwin}

1943 The Life and Art of Albrecht Dürer. Princeton, Princeton University Press.

\section{Peirce, Charles Sander}

1978 Écrits sur le signe. Rassemblés, traduits et commentés par $\mathrm{G}$. Deledalle. Paris, Seuil.

\section{Riegl, Alois}

2009 [1902] Le portrait de groupe dans la peinture hollandaise. Paris, Hazan.

\section{SeTtis, Salvatore}

2005 Iconografia dell'arte italiana : una linea. Turin, Einaudi.

\section{Severi, Carlo}

2003 «Warburg anthropologue, ou le déchiffrement d'une utopie. De la biologie des images à l'anthropologie de la mémoire », L'Homme 165 (numéro spécial «Image et anthropologie », Carlo Severi [éd.]] : ?7-129.

2007 Le Principe de la chimère. Une anthropologie de la mémoire. Paris, Éditions rue d'Ulm-musée du quai Branly [«Asthetica »].

2009 « L'univers des arts de la mémoire. Anthropologie d'un artefact mental », Annales HSS 2 : 463-493.

2010 «La parole prêtée. Comment parlent les images », in Carlo Severi et Julien Bonhomme (dir.), Paroles en actes. Paris, L’Herne [«Cahiers d'anthropologie sociale 5 ») : 11-41.

\section{SuIDA, William}

1953 Bramante pittore e Bramantino. Milano, Ceschina.

\section{SHEARMAN, John}

1992 Only Connect: Art and the Spectator in the Italian Renaissance. Princeton, Princeton University Press.

\section{Velthem, Lúcia Hussak van}

20030 Belo é a Fera. A estética da produção e da predação entre os Wayana. Lisbonne, Museu Nacional de Etnologia.

WARBURG, Aby

2010 «Du laboratoire au musée », in Marc-Olivier Wahler et Frédéric Grossi [dir.], Du yodel à la physique quantique. Vol. IV. Paris, Palais de Tokyo.

\section{WiLBERT, Johannes}

1981 «Warao Cosmology and Yekwana Roundhouse Symbolism », Journal of Latin American Lore ? (1) : 37-?2.

\section{Winkelmann, Johann Joachim} 1973 II bello nell'arte. Turin, Einaudi.

\section{WitTKOWER, Rudolf}

1977 « Hieroglyphics in the Early Renaissance », Allegory and the migration of symbols. Londres, Thames \& Hudson : 113-128. 


\section{Résumé / Abstract}

Carlo Severi, L'espace chimérique. Perception et projection dans les actes de regards - Le concept de représentation chimérique, que nous avons proposé dans le cadre d'une théorie anthropologique de la mémoire, a suscité autant de débats que de recherches nouvelles. Trois types de questions ont été posés, qui concernent la morphologie, la logique et le type d'appréhension esthétique, ou acte de regard, qui caractérisent cette notion.

Répondre à ces questionnements permet de passer de la définition d'une typologie des représentations, à l'identification d'une logique des relations représentées par l'image. Pour ce faire, nous essayons d'abord de préciser la définition de la notion de chimère du point de vue morphologique, logique et esthétique. Nous essayons ensuite de montrer comment cette nouvelle approche permet d'interpréter deux traditions iconographiques amazoniennes: les vanneries yekwana et wayana.
Carlo Severi, Chimeric Space. Perception and Projection in "Gaze Acts" - The concept of chimeric representation outlined in my theory of memory not only opened up new avenues of research, but also provoked considerable debate. Three main sorts of question were raised, concerning the morphology, the logic and the form of aesthetic apprehension, or "gaze act", associated with this concept.

In responding to these questions, this article moves away from outlining a typology of representations and instead focusses on identifying the specific relational logic inherent in images. To this end, it first seeks to define the chimera from a morphological, logical and aesthetic point of view, before showing how this new approach can be used to interpret two Amazonian iconographic traditions: Ye'kuana and Wayana basket-weaving. 